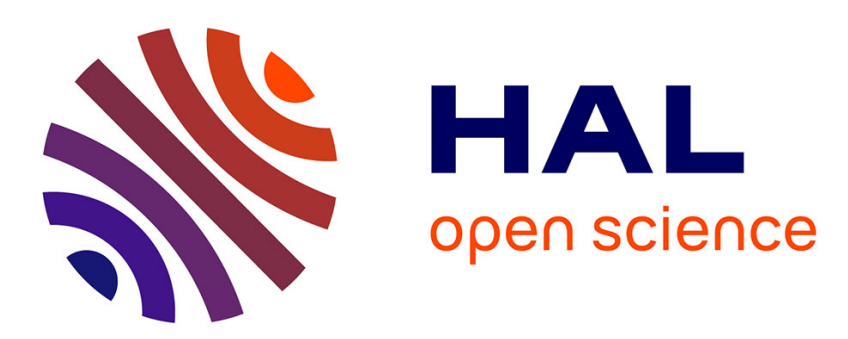

\title{
Reliability Assessment of Systems Subject to Dependent Degradation Processes and Random Shocks
}

\author{
Yan-Hui Lin, Yan-Fu Li, Enrico Zio
}

\section{To cite this version:}

Yan-Hui Lin, Yan-Fu Li, Enrico Zio. Reliability Assessment of Systems Subject to Dependent Degradation Processes and Random Shocks. IIE Transactions, 2016, 10.1080/0740817X.2016.1190481. hal-01342101

\section{HAL Id: hal-01342101 \\ https://hal.science/hal-01342101}

Submitted on 7 Jul 2020

HAL is a multi-disciplinary open access archive for the deposit and dissemination of scientific research documents, whether they are published or not. The documents may come from teaching and research institutions in France or abroad, or from public or private research centers.
L'archive ouverte pluridisciplinaire HAL, est destinée au dépôt et à la diffusion de documents scientifiques de niveau recherche, publiés ou non, émanant des établissements d'enseignement et de recherche français ou étrangers, des laboratoires publics ou privés. 


\title{
Reliability Assessmentof SystemsSubject to Dependent Degradation Processes and Random Shocks
}

\author{
Yan-Hui Lin ${ }^{1,2}$, Yan-Fu Li ${ }^{1}$, Enrico Zio ${ }^{1,3}$ \\ ${ }^{1}$ Chaire Systems Science and the Energy Challenge, Fondation Electricité de France (EDF) \\ Laboratoire Genie Industriel, CentraleSupélec, Université Paris-Saclay Grande voie des Vignes, \\ 92290 Chatenay-Malabry, France \\ ${ }^{2}$ Department of Systems Engineering and Engineering Management, City University of Hong Kong, Hong \\ Kong, China \\ ${ }^{3}$ Politecnico di Milano, Italy
}

\begin{abstract}
System failures can be induced by internal degradation mechanisms or by external causes. In this paper, we consider the reliability ofsystems experiencing both degradation and random shock processes. The dependencies between degradation processes and random shocks, and among degradation processes areexplicitly modelled. The degradation processes of system components are modeled by multi-state models (MSMs) and physics-based models (PBMs).The piecewisedeterministic Markov process modeling framework is employed to combine MSMs and PBMs, and for incorporating degradation and random shocks dependencies. The Monte Carlo simulation and finite-volume methodsare used to compute the system reliability. Asubsystem of a residual heat removal system in a nuclear power plant is considered as illustrative case.
\end{abstract}

Key Words -multi-state system, system reliability assessment, degradation, random shocks, dependency, piecewise-deterministic Markov process, Monte Carlo simulation, residual heat removal system.

\section{Acronyms}

PBMs

Physics-based models

MSMs

Multi-state models

PDMP

Piecewise-deterministic Markov process

MCS

Monte Carlo simulation 
K

$L$

$\boldsymbol{S}_{K_{n}}$

$Y_{K_{n}}(t)$

$\lambda_{i}\left(j \mid \boldsymbol{\theta}_{K_{n}}\right)$

$\mathcal{F}_{K_{n}}$

$\boldsymbol{X}_{L_{m}}(t)$

$\boldsymbol{X}_{L_{m}}^{\boldsymbol{D}}(t)$

$\boldsymbol{X}_{L_{m}}^{P}(t)$

$\mathcal{F}_{L_{m}}$

$N(t)$

$\mu$

$Y_{K_{n}}^{\prime}(t)$

$\mathcal{F}_{K_{n}}^{\prime}$

$W_{i}$

D

$\boldsymbol{H}_{i}$

$N^{c}(t)$

$\boldsymbol{D}_{L_{m}}$
Finite-volume

Residual heat removal system

\section{Notations}

Group of degradation processes modeled by MSMs

Group of degradation processes modeled by PBMs

Finite state set of degradation $\operatorname{process} K_{n}$

State variable of degradation process $K_{n}$

Transition rate from state $i$ to $j$

Set of failure states of degradation process $K_{n}$

Time-dependent continuous variables of degradation process $L_{m}$

Non-decreasing degradation variables vector

Physical variables vector

Set of failure states of degradation process $L_{m}$

Number of random shocks occurred until time $t$

Arrival rate of random shock process

Degradation level of $K_{n}$ considering random shocks

Set of failure states of $Y_{K_{n}}^{\prime}(t)$

Shock load of the $i$-th shock

Maximal material strength

Instantaneous random increase caused by the $i$-th cumulative shock

Number of cumulative shocks occurred until time $t$

Degradation level of $L_{m}$ considering random shocks 


$\begin{array}{ll}\boldsymbol{Y}(t) & \text { Degradation state of the processes of set } \boldsymbol{K} \\ \boldsymbol{X}(t) & \text { Degradation state of the processes of set } \boldsymbol{L} \\ \boldsymbol{\theta}_{\boldsymbol{K}} & \text { Environmental and operational factors in } \boldsymbol{K} \\ \boldsymbol{\theta}_{\boldsymbol{L}} & \text { Environmental and operational factors in } \boldsymbol{L} \\ \boldsymbol{Z}(t) & \text { Degradation process of the system } \\ \boldsymbol{Y}^{\prime}(t) & \text { Degradation state of the processes of set } \boldsymbol{K} \text { considering random shocks } \\ \boldsymbol{X}^{\prime}(t) & \text {-th jump time in } \boldsymbol{Y}^{\prime}(t) \\ T_{k} & \text { Deterministic physics equations of } \boldsymbol{X}(t) \\ \boldsymbol{Z}_{k}=\left(\boldsymbol{X}_{k}^{\prime}, \boldsymbol{Y}_{k}^{\prime}\right) \text { State of } \boldsymbol{Z}(t), \boldsymbol{X}^{\prime}(t), \boldsymbol{Y}^{\prime}(t) \text { afterk-th jump of } \boldsymbol{Y}^{\prime}(t) \\ \boldsymbol{f}_{\boldsymbol{L}}^{\boldsymbol{Y}^{\prime}(t)}\left(\boldsymbol{X}(t) \mid \boldsymbol{\theta}_{\boldsymbol{L}}\right) & \text { Probansition rate of } \boldsymbol{Y}^{\prime}(t) \text { from state } \boldsymbol{y}_{i} \text { to } \boldsymbol{y}_{j} \\ \lambda_{\boldsymbol{y}_{i}, \boldsymbol{y}_{j}}\left(\boldsymbol{x} \mid \boldsymbol{\theta}_{\boldsymbol{K}}\right) & \text { Semi-Markov kernel of }\left\{\boldsymbol{Z}_{n}, T_{n}\right\}_{n \geq 0} \\ N\left(\boldsymbol{i},\left(d \boldsymbol{x}, \boldsymbol{y}_{j}\right), d t\right) & \text { considering random shocks } \\ d F_{\boldsymbol{i}}(t) & \end{array}$

\section{INTRODUCTION}

The evaluation of the system reliability over time is an important and critical task. For example, the reliability of safety systems in nuclear power plants, such as reactor shutdown, emergency core cooling systems and other safety multi-component systems in nuclear industry, need to meet safety requirements imposed by regulation to ensure their operational safety (Marseguerra, et al., 2004). The instances when the requirements are not satisfied can be identified byreliability analysis. Reliability improvement actions can, then, be performed by design on maintenance, to avoid possible human and economic losses. In this paper, we investigate the reliability assessment of multi-component systems subject to dependent degradation processes influenced by random shocks. The dependencies pose challenging issues in system reliability modeling and assessment (Schöttl, 1996) (e.g. for microelectromechanical systems, which are complex systems experiencing dependent component failure processes and multiple dependent competing failure processes for each component (Song, et al., 2014b)). System failures can be induced byinternal degradation mechanisms (e.g. wear, fatigue and erosion)orby external causes(e.g. thermal and mechanical shocks)(Jiang, et al., 2015). The 
interactions between these factors needto be considered under certain circumstances, e.g. whendegradation processes andrandom shocksare $s$-dependent (e.g. single-event overloadswith safe shock magnitudescan influence the fatigue crack growth of stents by causing instantaneous increase on the crack propagation(Keedy and Feng, 2013)), orthe degradation state of some components in onesystem can influence the degradation dynamics or the remaining useful life of the others(e.g. the degradation of the pre-filtration stations leading to a lower performance level of the sand filter in a water treatment plant(Rasmekomen and Parlikad, 2013)). Neglecting these aspects may result in overestimation of system reliability (Wang and Pham, 2012).

In industrial systems, many critical components (e.g. valves and pumps in the nuclear and aerospace industries) are designed to be highly reliable, for which statistical degradation/failure data are often limited. In this case, multi-state models (MSMs) (Black, et al., 2005, Giorgio, et al., 2011, Moghaddass and Zuo, 2014) and physics-based models (PBMs) (Chookah, et al., 2011, Daigle and Goebel, 2011, Wang, et al., 2014) can be used to describe the evolution of degradation in components and systems. A MSM describes the degradation process in a discrete way, supported by material science knowledge (Lin, et al., 2015b) and/or available but limited degradation/failure historical data from field collection or degradation tests (Giorgio, et al., 2011). On the contrary, a PBM gives an integrated mechanistic description of the component life consistent with the underlying real degradation mechanisms under operating conditions (Hu, et al., 2014), by using physics knowledge modeled by corresponding mathematical equations (Chookah, et al., 2011). In practice, degradation models of different nature have to be applied depending on the available information of the degradation processes. Recently, the piecewise-deterministic Markov process (PDMP) modeling framework has been employed to incorporate PBMs and MSMs, and to treat the dependencies among degradation processes but without considering the influences of random shocks(Lin, et al., 2015a). On the other hand, random shocks can accelerate the degradation processes (e.g. internal thermal shocks and water hammers onto power plant components (Salonen, et al., 2007)).

The reliability of systems experiencing both degradation and random shocks is a problem that has been widely studied(Becker, et al., 2002, Jiang, et al., 2012, Lin, et al., 2015b, Peng, et al., 2010, Rafiee, et al., 2014, Song, et al., 2014a, Song, et al., 2014b, Wang and Pham, 2012, Yang, et al., 2011).The dependency among these processes leading to failure has posed some challengesto reliability modeling.A literature review is presented below, to position our contributions within the existing works. Previous research has focused on the dependency between one type of degradation processes (continuous or multi-state) and random shocks.For continuous degradation processes,Peng, et al. (2010)considered systems with one lineardegradation path where shocks can bring additional abrupt degradation damage if the shock loads donot exceed the maximum strength of the material;multi-component systems subject to multiple linear degradation paths have been further considered bySong, et al. (2014b); Jiang, et al. (2012)considered changes in themaximal strength of the material when systems are deteriorating under different situations;Becker, et al. (2002)extended 
the theory of dynamic reliability to incorporate random changes of the degradation variables due to random shocks; Ye, et al. (2011)considered the destructive power of a shock depending not only on the shock magnitude but also on the state of the system;Wang, et al. (2011)considered two types of the effects of shocks: a sudden increase in the failure rate after a shock and a direct random change in the degradation after the occurrence of a shock;Rafiee, et al. (2014)proposed reliability models for systems for whichthe degradation path has achanging degradation rate according to particular random shock patterns;Song, et al. (2014a)studied random shocks with specific sizes or functions, which can selectively affect the degradation processes of one or more components (not necessarily all components) in one system.For multi-state degradation processes, Yang, et al. (2011)combined random shocks with Markov degradation models where shocks can lead the systems to further degraded states;Lin, et al. (2015b)integrated random shocks into multi-state physics models of degradation processeswhere the influences of shocks are dependent on the current degradation condition; Ruiz-Castro (2016)considered external shocks which could produce several effects; extreme failure, cumulative damage and when the damage reaches a threshold state, a non-repairable failure occurs, and changes in the internal performance of the device. Note that no work has considered systems with both continuous and multi-state degradation processes and subject to random shocks, and few studies have explicitly considered both the dependencies between degradation processes and random shocks, and among degradation processes themselves.Wang and Pham (2012)employed copulasto handle these two types of dependencies; however,sufficient degradation/failure data is required to determine the copula functionsthrough statistical inference.

In this paper, we extend the PDMP modeling framework for system reliability assessment, considering not only the dependencies among degradation processes but also the impacts of random shocks. To the best knowledge of the authors, this is the first work investigating systems with both continuous and multi-state degradation processes, subject to random shocks and considering the dependencies between degradation processes and random shocks, and among degradation processes. Since the analytical solution is difficult to obtain due to thecomplexity of the system being considered, we employ two numerical approaches to assess system reliability: the Monte Carlo (MC) simulation(Marseguerra and Zio, 1996) and the finite-volume (FV)(Cocozza-Thivent, et al., 2006) methods.

The remainder of this article is organized as follows. Section 2 provides the assumptions and descriptions of the degradation processes and random shocks. Section 3 presents the extended modelfor systems with degradation and random shock processes, consideringtheir dependencies. The proposed MC simulationand FV methods are presented in Section 4. Section 5 presents an illustrative study taken from a real residual heat removal system (RHRS) operated by Électricité de France (EDF). The RHRS is used for cooling the reactor during and following shutdown, contributing to safety by removing heat from the core and transferring it to the environment. Specifically, we consider an important subsystem consisting of a pneumatic valve and a centrifugal pump in series. This system 
setting is widely used, under different conditions, in a variety of domains for fluid delivery (from water supply to spacecraft fueling systems) (Daigle and Goebel, 2011, Daigle and Goebel, 2013). Numerical results and analysis are presented in Section 6. Section 7 concludes the work.

\section{ASSUMPTIONS AND MODEL DESCRIPTIONS}

We consider a multi-component system. Each component may be affected by multiple degradation mechanisms or processes, possibly dependent. The degradation processes can be separated into two groups: (1) $\boldsymbol{K}$ consists ofprocesses fit to be modeled by MSMs; (2) $\boldsymbol{L}$ consists ofprocesses fit to be modeled by PBMs.

\subsection{Degradation models}

\subsubsection{MSMs}

We follow the assumptions on MSMs made in Lin, et al. (2014):

- A degradation process, $Y_{K_{n}}(t), K_{n} \in \boldsymbol{K}$ of group (1), takes values from a finite state set denoted by $\boldsymbol{S}_{K_{n}}=\{0,1, \ldots, d\}$, where $d$ is the perfect functioning state and 0 is the complete failure state. The component is functioning or partially functioning in the intermediate degradation states. The transition rates $\lambda_{i}\left(j \mid \boldsymbol{\theta}_{K_{n}}\right), \forall i, j \in \boldsymbol{S}_{K_{n}}, i>j$ characterize the degradation transition probabilities from state $i$ to state $j$, where $\boldsymbol{\theta}_{K_{n}}$ represents the environmental factors relevant to $K_{n}$ and the related parameters of $\lambda_{K_{n}}$. We follow the assumption of Markov property made inGiorgio, et al. (2011), Yeh (1997) and Zuo, et al. (2000); Markov processes are widely used to describe components degradation processes. The transition rates between different degradation states are estimated from the degradation and/or failure data from historical field collection. The failure state set of the process $K_{n}$ is denoted by $\mathcal{F}_{K_{n}}=\{0\}$.

\subsubsection{PBMs}

We follow the assumptions on PBMs made in Lin, et al. (2014):

- A degradation process $\boldsymbol{X}_{L_{m}}(t), L_{m} \in \boldsymbol{L}$ of group (2), has $d_{L_{m}}$ time-dependent continuous variables, whose evolution is characterized by a system of first-order differential equations $\boldsymbol{X}_{L_{m}}^{\cdot}(t)=\boldsymbol{f}_{L_{m}}\left(\boldsymbol{X}_{L_{m}}(t), t \mid \boldsymbol{\theta}_{L_{m}}\right)$, i.e. physics equations, where $\boldsymbol{\theta}_{L_{m}}$ represents the environmental factors influential to $L_{m}$ (e.g. temperature and pressure) and the parameters used in $\boldsymbol{f}_{L_{m}}$. This assumption is made in Lorton, et al. (2013) and widely used (Daigle and 
Goebel, 2011, Daigle and Goebel, 2013). Note that higher-order differential equations can be converted into a system of first-order differential equations by introducing extra variables(Zwillinger, 1998).

- $\quad \boldsymbol{X}_{L_{m}}(t)=\left(\boldsymbol{X}_{L_{m}}^{\boldsymbol{D}}(t), \boldsymbol{X}_{L_{m}}^{\boldsymbol{P}}(t)\right)$ contains: (1) the non-decreasing degradation variables $\boldsymbol{X}_{L_{m}}^{\boldsymbol{D}}(t)$ (e.g. leak area) describing the degradation process, where $\boldsymbol{D}$ is the set of degradation variables indices (the same assumption has been already widely used(Daigle and Goebel, 2011, Daigle and Goebel, 2013, Keedy and Feng, 2013)); (2) the physical variables $\boldsymbol{X}_{L_{m}}^{\boldsymbol{P}}(t)$ (e.g. velocity and force), which influence $\boldsymbol{X}_{L_{m}}^{\boldsymbol{D}}(t)$, where $\boldsymbol{P}$ is the set of physical variable indices. The generic degradation process $L_{m}$ reaches failure when one $x_{L_{m}}^{i}(t) \in \boldsymbol{X}_{L_{m}}^{\boldsymbol{D}}(t)$ reaches or exceeds its corresponding failure threshold denoted by $x_{L_{m}}^{i}{ }^{*}$. The failure state set of the process $L_{m}$ is denoted by $\mathcal{F}_{L_{m}}$.

\subsection{Random shocks}

Random shocks can influence the degradation processes of the components. The following assumptions are made, similarly to various previous works(Jiang, et al., 2012, Peng, et al., 2010, Rafiee, et al., 2014, Song, et al., 2014a, Yang, et al., 2011).

- Random shocks occur in time according to a homogeneous Poisson process $\{N(t), t \geq 0\}$ with constant arrival rate $\mu$ (Fig. 1), where the random variable $N(t)$ denotes the number of random shocks occurred until time $t$.

- The damages of random shocks aredivided into two types: extreme and cumulative.

- Extreme and cumulative shocks are mutually exclusive.

- Extreme shocksimmediately lead the componentsto failure, whereas cumulative shocks gradually deteriorate the components.

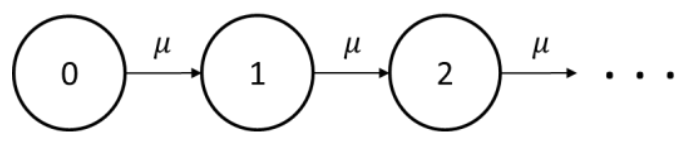

Fig. 1. Random shock process

\section{DEPENDENT DEGRADATION PROCESSES AND RANDOM SHOCKS}

\subsection{Dependency between degradation processes and random shocks}

Due to the different nature of PBMs and MSMs, the impacts of random shocks on the two groups 
of components are characterized in different ways.

\subsubsection{Impacts on MSMs}

In the generic degradation process $K_{n} \in \boldsymbol{K}$, random shocks can cause the process variable $Y_{K_{n}}(t)$ to step from state $i$ to a further degraded state $j$ with probability $p_{i j}, i>j$ (Yang, et al., 2011), with $p_{i 0}$ denoting the probability that the random shockis extreme, i.e. leading to failure state 0 upon occurrencefrom state $Y_{K_{n}}(t)=i$. By combining the original degradation and the random shock processes, the resulting process $\boldsymbol{Y}_{K_{n}}^{\prime}(t)$ is a homogeneous continuous-time Markov chain of the type depicted in Fig. 2. Each layer indicates one degradation state of $Y_{K_{n}}(t)$, and the numbers in each layer indicate the numbers of shocks experienced up to time $t$ in the process $K_{n}$, denoted by $k$. The state of $\boldsymbol{Y}_{K_{n}}^{\prime}(t)$ is, then, represented by thepair $\left(Y_{K_{n}}(t), k\right)$. The transitions represented by solid lines are due to the original degradation process, characterized by the original transition rates, which do not influence the value of $k$. The transitions represented by dotted lines are due to random shocks, which cause $k$ to be increased by one. $\mu p_{i j}, i>j$ is the rate of occurrence of a shock which will cause the process stepping to the $j$-th layer from the $i$-th layer. Note that $K_{n}$ fails whenever $Y_{K_{n}}(t)$ reaches the degradation state 0 , no matter how many shocks it has experienced. Therefore, the space of the failure states of $\boldsymbol{Y}_{K_{n}}^{\prime}(t)$ is denoted by $\mathcal{F}_{K_{n}}^{\prime}=\{(0, b), \forall b \in \mathbb{N}\}$. The state space of $\boldsymbol{Y}_{K_{n}}^{\prime}(t)$ is denoted by $\boldsymbol{S}_{K_{n}}^{\prime}=\left\{(a, b), \forall a \in \boldsymbol{S}_{K_{n}}, b \in \mathbb{N}\right\}$.

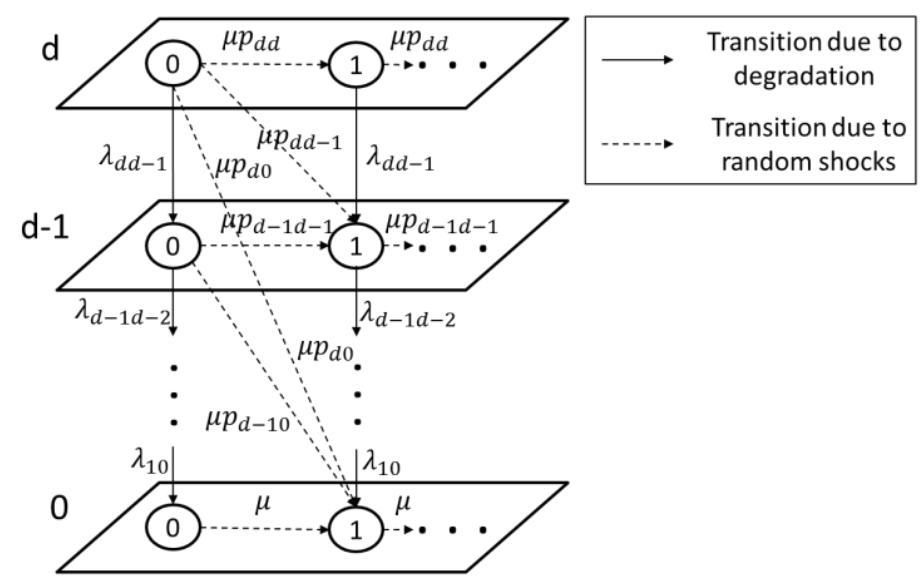

Fig. 2. Degradation process $K_{n}$ and random shocks.

\subsubsection{Impacts on PBMs}

In the generic degradation process $L_{m} \in \boldsymbol{L}$, the $i$-th shock becomes extreme if the shock load $W_{i}$ exceeds the maximal material strength $D$, otherwise, it can bring an instantaneous random increase $\boldsymbol{H}_{i}$ 
to $\boldsymbol{X}_{L_{m}}(t)$ (Song, et al., 2014b). The overall degradation level of $L_{m}$ is expressed as follows:

$$
\boldsymbol{D}_{L_{m}}(t)=\left\{\begin{array}{c}
\boldsymbol{X}_{L_{m}}(t)+\sum_{i=1}^{N^{c}(t)} \boldsymbol{H}_{i}, \text { if } N^{\prime}(t) \neq 0 \\
\boldsymbol{X}_{L_{m}}(t), \quad \text { if } N^{\prime}(t)=0
\end{array}\right.
$$

where $N^{c}(t)$ is the number of cumulative shocks occurred in the developing $L_{m}$ process before the extreme shock occurs until time $t$. The process $L_{m}$ leads to failure if $\boldsymbol{D}_{L_{m}}(t)$ reaches the predefined failure state set $\mathcal{F}_{L_{m}}$ or a shock with load larger than $D$ occurs. An example of degradation process $L_{m}$ considering random shocks is shown in Fig. 3 , where $W_{i}$ is the shock load of the $i$-th shock occurred at time $t_{i}, i=1,2,3$.The center figure in Fig. 3 represents the evolution of the physical variable (e.g. velocity and force), which can influence the degradation variable (top figure)and may also be influenced by random shocks (bottom figure).

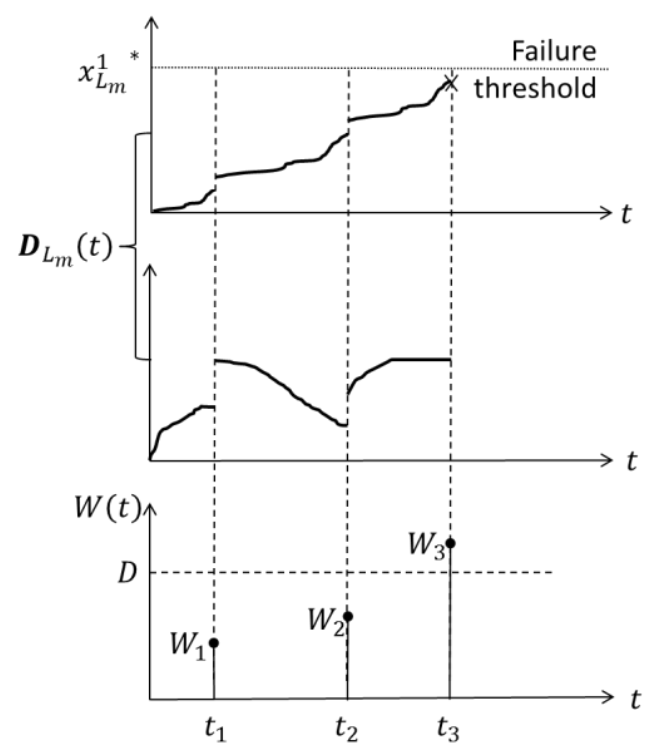

Fig. 3. An example of degradation process $L_{m}$ with random shocks. Top Figure:degradation variable; Center Figure: physical variable; Bottom Figure: random shock process.

\subsection{Dependency among degradation processes}

Dependencies may exist among degradation processes within each group and between the two groups. The degradation states of the processes of set $K$ may influence the evolution of the continuous variablesof the degradation processes of set $\boldsymbol{L}$, andthe degradation levels ofthe lattermay influence the transition times and transition directions of the former(the detailed formulations are shown in eqs. (2) and (3)) (Lin, et al., 2015a).

Let $\boldsymbol{Y}(t)=\left(Y_{K_{1}}(t), \ldots, Y_{K_{N}}(t)\right) \in \boldsymbol{S}=\left\{0,1, \ldots, d_{\boldsymbol{S}}\right\}$ and $\boldsymbol{X}(t)=\left(\boldsymbol{X}_{L_{1}}(t), \ldots, \boldsymbol{X}_{L_{M}}(t)\right) \in \mathbb{R}^{d_{L}}$. The evolution of $\boldsymbol{Y}(t)$ is governed by the transition rates which depend on the states of the degradation processes in the first group $\boldsymbol{X}(t)$ and also in the second group $\boldsymbol{Y}(t)$, as follows: 


$$
\begin{gathered}
\lim _{\Delta t \rightarrow 0} P\left(\boldsymbol{Y}(t+\Delta t)=\boldsymbol{j} \mid \boldsymbol{X}(t), \boldsymbol{Y}(t)=\boldsymbol{i}, \boldsymbol{\theta}_{\boldsymbol{K}}=\bigcup_{n=1}^{N} \boldsymbol{\theta}_{K_{n}}\right) / \Delta t \\
=\lambda_{\boldsymbol{i}}\left(\boldsymbol{j} \mid \boldsymbol{X}(t), \boldsymbol{\theta}_{\boldsymbol{K}}\right), \forall t \geq 0, \boldsymbol{i}, \boldsymbol{j} \in \boldsymbol{S}, \boldsymbol{i} \neq \boldsymbol{j}
\end{gathered}
$$

The evolution of $\boldsymbol{X}(t)$ is described by mathematical equations representing the underlying physics and depends on the states of the degradation processes in the second group $\boldsymbol{Y}(t)$ and also in the first group $\boldsymbol{X}(t)$, as follows:

$$
\begin{aligned}
\dot{\boldsymbol{X}}(t)=\left(\boldsymbol{X}_{L_{1}}(t), \ldots, \boldsymbol{X}_{L_{M}}^{\cdot}(t)\right)=\left(\boldsymbol{f}_{L_{1}}{ }^{\boldsymbol{Y}(t)}\left(\boldsymbol{X}(t), t \mid \boldsymbol{\theta}_{L_{1}}\right), \ldots, \boldsymbol{f}_{L_{M}}{ }^{\boldsymbol{Y}(t)}\left(\boldsymbol{X}(t), t \mid \boldsymbol{\theta}_{L_{M}}\right)\right) \\
=\boldsymbol{f}_{\boldsymbol{L}}{ }^{\boldsymbol{Y}(t)}\left(\boldsymbol{X}(t), t \mid \boldsymbol{\theta}_{\boldsymbol{L}}=\bigcup_{m=1}^{M} \boldsymbol{\theta}_{L_{m}}\right)
\end{aligned}
$$

\subsection{PDMPs for systems subject to degradation dependency and random shocks}

Let $\boldsymbol{Z}(t)$ denote the overall degradation process of the system:

$$
\boldsymbol{Z}(t)=\left(\boldsymbol{X}^{\prime}(t)=\left(\boldsymbol{D}_{L_{1}}(t), \ldots, \boldsymbol{D}_{L_{M}}(t)\right), \boldsymbol{Y}^{\prime}(t)=(\boldsymbol{Y}(t), N(t))\right) \in \boldsymbol{E}=\mathbb{R}^{d_{L}} \times \boldsymbol{S}^{\prime}(4)
$$

where $\boldsymbol{E}$ is a space combining $\mathbb{R}^{d_{L}}$ and $\boldsymbol{S}^{\prime}=\boldsymbol{S} \times \mathbb{N}$.Let $T_{k}, k \in \mathbb{N}$ denote the $k$-th jump time in $\boldsymbol{Y}^{\prime}(t)$ and $\boldsymbol{Z}_{k}=\boldsymbol{Z}\left(T_{k}\right)=\left(\boldsymbol{X}^{\prime}\left(T_{k}\right), \boldsymbol{Y}^{\prime}\left(T_{k}\right)\right)=\left(\boldsymbol{X}_{k}^{\prime}, \boldsymbol{Y}_{k}^{\prime}\right)$. The evolution of $\boldsymbol{Z}(t)$ between two consecutive jumps of $\boldsymbol{Y}^{\prime}(t)$, between which no shock occurs to the system and the degradation state does not change, can be written as follows:

$$
\begin{aligned}
& \dot{\boldsymbol{Z}}(t)=\left(\dot{\boldsymbol{X}}^{\prime}(t), \dot{\boldsymbol{Y}}^{\prime}(t)\right) \\
& =\left(\boldsymbol{f}_{\boldsymbol{L}}{ }^{\boldsymbol{Y}^{\prime}(t)}\left(\boldsymbol{X}(t) \mid \boldsymbol{\theta}_{\boldsymbol{L}}\right),(\mathbf{0}, 0)\right), \text { for } t \in\left[T_{k}, T_{k+1}[(5)\right.
\end{aligned}
$$

According to the definition inCocozza-Thivent (2011), $\boldsymbol{Z}(t)$ is a PDMP since (1)it can be written as $\boldsymbol{Z}(t)=\varphi\left(\boldsymbol{Z}_{k}, t-T_{k}\right)$, for $t \in\left[T_{k}, T_{k+1}[\right.$ and $\varphi$ satisfies $\varphi(\boldsymbol{y}, t+s)=\varphi(\varphi(\boldsymbol{y}, t), s), \forall t, s \geq 0, \boldsymbol{y} \in$ $\boldsymbol{E}$, and $t \rightarrow \varphi(\boldsymbol{y}, t), \forall t \geq 0, \boldsymbol{y} \in \boldsymbol{E}$ is right continuous with left limits and (2) $\left\{\boldsymbol{Z}_{n}, T_{n}\right\}_{n \geq 0}$ is a Markov renewal process defined on the space $\boldsymbol{E} \times \mathbb{R}^{+}$. The probability that $\boldsymbol{Z}(t)$ will step to state $\boldsymbol{j}$ from state $\boldsymbol{Z}_{k}$ in the time interval $\left[T_{k}, T_{k}+t\right]$, given $\left\{\boldsymbol{Z}_{i}, T_{i}\right\}_{i \leq k}$ is as follows:

$$
\begin{array}{r}
P\left[\boldsymbol{Z}_{k+1}=\boldsymbol{j}, T_{k+1} \in\left[T_{k}, T_{k}+t\right] \mid\left\{\boldsymbol{Z}_{i}, T_{i}\right\}_{i \leq k}\right]=P\left[\boldsymbol{Z}_{k+1}=\boldsymbol{j}, T_{k+1} \in\left[T_{k}, T_{k}+t\right] \mid \boldsymbol{Z}_{k}\right], \\
\forall k \in \mathbb{N}, \boldsymbol{j} \in \boldsymbol{E}, \boldsymbol{j} \neq \boldsymbol{Z}_{k}
\end{array}
$$

$\left\{\boldsymbol{Z}_{n}, T_{n}\right\}_{n \geq 0}$ is characterized by the semi-Markov kernel $N\left(\boldsymbol{i}=\left(\boldsymbol{x}_{i}, \boldsymbol{y}_{i}\right),\left(d \boldsymbol{x}, \boldsymbol{y}_{j}\right), d t\right)=P\left[\boldsymbol{X}_{k+1}^{\prime} \in\right.$ $\left.[\boldsymbol{x}, \boldsymbol{x}+d \boldsymbol{x}], \boldsymbol{Y}_{k+1}^{\prime}=\boldsymbol{y}_{j}, T_{k+1}-T_{k} \in[t, t+d t] \mid \boldsymbol{Z}_{k}=\boldsymbol{i}\right], \forall k \in \mathbb{N}, \boldsymbol{y}_{i}, \boldsymbol{y}_{j} \in \boldsymbol{S}^{\prime}, \boldsymbol{x}_{i}, d \boldsymbol{x} \in \mathbb{R}^{d_{L}}, d \boldsymbol{x} \rightarrow$

$\mathbf{0}, d t \rightarrow 0$, which can be reformulated as follows:

$$
\begin{gathered}
N\left(\boldsymbol{i}=\left(\boldsymbol{x}_{i}, \boldsymbol{y}_{i}\right),\left(d \boldsymbol{x}, \boldsymbol{y}_{j}\right), d t\right) \\
=P\left[\boldsymbol{X}_{k+1}^{\prime} \in[\boldsymbol{x}, \boldsymbol{x}+d \boldsymbol{x}], \boldsymbol{Y}_{k+1}^{\prime}=\boldsymbol{y}_{j} \mid T_{k+1}-T_{k} \in[t, t+d t], \boldsymbol{Z}_{k}=\boldsymbol{i}\right] \\
\cdot P\left[T_{k+1}-T_{k} \in[t, t+d t] \mid \boldsymbol{Z}_{k}=\boldsymbol{i}\right] \\
=Q\left(\varphi(\boldsymbol{i}, t),\left(d \boldsymbol{x}, \boldsymbol{y}_{j}\right)\right) d F_{\boldsymbol{i}}(t)(7)
\end{gathered}
$$

where $Q\left(\varphi(\boldsymbol{i}, t),\left(d \boldsymbol{x}, \boldsymbol{y}_{j}\right)\right)$ is the probability distribution of state $\boldsymbol{Z}_{k+1}$ given $T_{k+1}-T_{k}=t$ and 
$\boldsymbol{Z}_{k}=\boldsymbol{i}$ and $d F_{\boldsymbol{i}}(t)$ is the probability distribution of $T_{k+1}-T_{k}$ given $\boldsymbol{Z}_{k}=\boldsymbol{i} \cdot Q\left(\varphi(\boldsymbol{i}, t),\left(d \boldsymbol{x}, \boldsymbol{y}_{j}\right)\right)$ can be reformulated as follows:

$$
\begin{gathered}
Q\left(\varphi\left(\boldsymbol{i}=\left(\boldsymbol{x}_{i}, \boldsymbol{y}_{i}\right), t\right),\left(d \boldsymbol{x}, \boldsymbol{y}_{j}\right)\right) \\
=P\left[\boldsymbol{X}_{k+1}^{\prime} \in[\boldsymbol{x}, \boldsymbol{x}+d \boldsymbol{x}], \boldsymbol{Y}_{k+1}^{\prime}=\boldsymbol{y}_{j} \mid T_{k+1}-T_{k} \in[t, t+d t], \boldsymbol{Z}_{k}=\boldsymbol{i}\right] \\
=P\left[\boldsymbol{X}_{k+1}^{\prime} \in[\boldsymbol{x}, \boldsymbol{x}+d \boldsymbol{x}] \mid \boldsymbol{Y}_{k+1}^{\prime}=\boldsymbol{y}_{j}, T_{k+1}-T_{k} \in[t, t+d t], \boldsymbol{Z}_{k}=\boldsymbol{i}\right] \\
\cdot P\left[\boldsymbol{Y}_{k+1}^{\prime}=\boldsymbol{y}_{j} \mid T_{k+1}-T_{k} \in[t, t+d t], \boldsymbol{Z}_{k}=\boldsymbol{i}\right](8)
\end{gathered}
$$

Let $p_{t}\left(d \boldsymbol{z}=\left(d \boldsymbol{x}, \boldsymbol{y}_{i}\right)\right)$ denote the probability distribution of $\boldsymbol{Z}(t)$, which obeys the ChapmanKolmogorov equation (Davis, 1993) as follows:

$$
\begin{aligned}
\int_{0}^{t} \sum_{\boldsymbol{y}_{i} \in \boldsymbol{S}^{\prime}} \int_{\mathbb{R}^{d_{L}}} \sum_{\boldsymbol{y}_{j} \in \boldsymbol{S}^{\prime}} \lambda_{\boldsymbol{y}_{i}, \boldsymbol{y}_{j}}\left(\boldsymbol{x} \mid \boldsymbol{\theta}_{\boldsymbol{K}}\right)\left(\int_{\mathbb{R}^{d_{L}}} \psi\left(\boldsymbol{y}_{j}, y\right) \mu\left(\boldsymbol{y}_{i}, \boldsymbol{y}_{j}, \boldsymbol{x}\right)(d \boldsymbol{y})-\psi\left(\boldsymbol{y}_{i}, \boldsymbol{x}\right)\right) p_{s}\left(d \boldsymbol{x}, \boldsymbol{y}_{i}\right) d s+ \\
\int_{0}^{t} \sum_{\boldsymbol{y}_{i} \in \boldsymbol{S}^{\prime}} \int_{\mathbb{R}^{d_{L}}} \boldsymbol{f}_{\boldsymbol{L}}^{\boldsymbol{y}_{i}}\left(\boldsymbol{x} \mid \boldsymbol{\theta}_{\boldsymbol{L}}\right) \operatorname{div}\left(\psi\left(\boldsymbol{y}_{i}, \boldsymbol{x}\right)\right) p_{s}\left(d \boldsymbol{x}, \boldsymbol{y}_{i}\right) d s- \\
\sum_{\boldsymbol{y}_{i} \in \boldsymbol{S}^{\prime}} \int_{\mathbb{R}^{d_{L}}} \psi\left(\boldsymbol{y}_{i}, \boldsymbol{x}\right) p_{t}\left(d \boldsymbol{x}, \boldsymbol{y}_{i}\right)+\sum_{\boldsymbol{y}_{i}^{\prime} \in \boldsymbol{S}^{\prime}} \int_{\mathbb{R}^{d_{L}}} \psi\left(\boldsymbol{y}_{i}, \boldsymbol{x}\right) p_{0}\left(d \boldsymbol{x}, \boldsymbol{y}_{i}\right)=0(9)
\end{aligned}
$$

where $\lambda_{\boldsymbol{y}_{i}, \boldsymbol{y}_{j}}\left(\boldsymbol{x} \mid \boldsymbol{\theta}_{\boldsymbol{K}}\right)$ is the transition rate of $\boldsymbol{Y}^{\prime}(t)$ from state $\boldsymbol{y}_{i}$ to $\boldsymbol{y}_{j}, \psi(\because)$ is any continuously differentiable function from $\boldsymbol{S}^{\prime} \times \mathbb{R}^{d_{L}}$ to $\mathbb{R}$ with a compact support and $\mu\left(\boldsymbol{y}_{i}, \boldsymbol{y}_{j}, \boldsymbol{x}\right)(d \boldsymbol{y})$ is the probability of $\boldsymbol{X}^{\prime}(t) \in[\boldsymbol{y}, \boldsymbol{y}+d \boldsymbol{y}]$ after jumping from $\boldsymbol{x}$ when $\boldsymbol{Y}^{\prime}(t)$ steps to state $\boldsymbol{y}_{j}$ from state $\boldsymbol{y}_{i}$.

The reliability of the system at time $t$ is defined as follows:

$$
R(t)=P[\boldsymbol{Z}(s) \notin \mathcal{F}, \forall s \leq t]=\int_{\mathbf{z} \notin \mathcal{F}} p_{t}(d \mathbf{z})(10)
$$

where $\mathcal{F}$ isthe space of the failure states of the system.

In the general modeling framework, the temporal variability is considered as follows: (1) the randomness in MSMs could imply the temporal variability of the degradation processes modeled by PBMs, as shown in Figs. (3) and (8); (2) in PBMs, the temporal variability of the degradation variables can be attributed to the time-varying physical variables associated with the underlying degradation mechanisms.

The parameters in the proposed model are mainly divided into three groups: (1) transition rates in multi-state models; (2) parameters in physics equations of physics-based models and (3) parameters characterizing random shock processes. The values of the first group can be estimated, using degradation and/or failure data from historical field collection or degradation tests, through maximum likelihood estimation for complete or incomplete data (Lisnianski, et al., 2010, Ogurtsova, 2014); they can also be estimated by domain experts using physics knowledge (e.g. the values of the transition rates in multi-state physics model (Lin, et al., 2015b)) are described by physics equations). For the second group, the laws of physics are used to build the equations describing the development of the underlying degradation mechanisms (e.g. fatigue, wear, corrosion, etc.) (Daigle and Goebel, 2011); the related parameter values can be estimated through regression models using degradation and/or failure data. For example, the physics equations of the fatigue cracking of the seal are built according 
to Paris-Erdogan law (Newby, 1991), which relates the stress intensity factor range to the crack growth under a fatigue stress regime; the values of the parameters are estimated through least squares regression methods by using data on crack length and cycles. The values of the third group can be estimated using related degradation and/or failure data obtained from historical field collection or shock tests (Chan and Englert, 2001), using likelihood-based inference or regression models (Ye, et al., 2011). For example, the Brown-Proschan model is employed to model wear and shock processes of tire treads (Ye, et al., 2011), whereby the likelihood function can be derived based on cumulative hazard function and the parameter values are estimated through maximum likelihood estimation.

\section{SYSTEM RELIABILITY ASSESSMENT UNDER DEPENDENT DEGRADATION AND RANDOM SHOCK PROCESSES}

The analytical solution of $R(t)$ is difficult to obtain mainly due to the complex PDMPs used to model the dependent degradation and random shock processes (Labeau, 1996). Therefore, we consider the following two approximate methods: the MC simulation method (Marseguerra and Zio, 1996) based on the semi-Markov kernel of $\left\{\boldsymbol{Z}_{n}, T_{n}\right\}_{n \geq 0}$ (eq. (7)) and the FV method (Cocozza-Thivent, et al., 2006) based on the Chapman-Kolmogorov equation (eq. (9)). They are two widely used approaches for solving PDMPs to evaluate reliability quantities. The FV method approximates the probability density function of PDMPs by discretizing the state space of the continuous variables and the time space. It is a method that can lead to comparable results as the MC simulation method, using less computing time for low dimensional problems (Eymard and Mercier, 2008).However, it is typically unsuited for high-dimensional problems or problems with complex equations describing the deterministic evolution. Besides, it is relatively more difficult to implement than the MC simulation method.

\subsection{MC simulation method}

The MC simulation method to compute the system reliability at time $t$ consists ofreplicating several times the lifeprocess of the system by repeatedly samplingitsholding time and arrival state from the corresponding probability distributions.Each replication continues until the time of system evolution reachest or until the system enters a state in the failure set $\mathcal{F}$.The procedure of the MC simulation method is as follows:

Set $N_{\text {max }}$ (the maximum number of replications) and $k=0$ (index of replication)

Set $k^{\prime}=0$ (number of replicationsthat end in a systemfailure state)

While $k<N_{\max }$

Initialize the system by setting $\boldsymbol{Z}=\left(\boldsymbol{X}^{\prime}(0), \boldsymbol{Y}^{\prime}(0)\right)$ (initial system state), and the time $T=0$ (initial system time) 
Set $t^{\prime}=0$ (state holding time)

While $T<t$

Sample a $t^{\prime}$ by using the probability distribution $d F_{Z}(t)$

Sample an arrival state $\boldsymbol{y}$ for stochastic process $\boldsymbol{Y}^{\prime}(t)$ and an arrival state $\boldsymbol{x}$ for process $\boldsymbol{X}^{\prime}(t)$ by using eq. (8)

$\operatorname{Set} T=T+t^{\prime}$
If $T \leq t$

Set $\boldsymbol{Z}=(\boldsymbol{x}, \boldsymbol{y})$

If $Z \in \mathcal{F}$

$$
\text { Set } k^{\prime}=k^{\prime}+1
$$

Break

End if

$\operatorname{Else}($ when $T>t$ )

If $\varphi\left(Z, t+t^{\prime}-T\right) \in \mathcal{F}$

Set $k^{\prime}=k^{\prime}+1$

Break

\section{End if}

\section{End if}

\section{End While}

Set $k=k+1$

\section{End While $\square$}

The estimated system reliability at time $t$ can be obtained by

$$
\widehat{R_{M C}}(t)=1-k^{\prime} / N_{\text {max }}
$$

where $k^{\prime}$ represents the number of trials that end in the failure state of the system, and the sample variance (Lewis and Böhm, 1984) is:

$$
\operatorname{var}_{\widehat{R_{M C}}}(t)=\widehat{R_{M C}}(t)\left(1-\widehat{R_{M C}}(t)\right) /\left(N_{\text {max }}-1\right)
$$

The MC simulation method is widely used in practice to evaluate system reliability(Zio, 2013). It is based on the strong law of large numbers and the central limit theorem and provides an unbiased estimator(Zio, 2013). The error on the estimate can be controlled within a confidence interval built based on the sample variance given in eq. (12), which can guarantee the consistency of the estimate. The accuracy of the MC simulation method increases as the number of replications increases. The MC simulation method is efficient in solving high-dimensional problems, since the sample variance does not depend on the number of dimensions. There are techniques to further improve the efficiency of MC simulation method (such as importance sampling, sequential MC, linear sampling, subset sampling, etc.)(Zio, 2013), which have to be designed according to the specific problems and have not been considered in our general reliability assessment framework. 


\subsection{FV method}

The FV method is an alternative for theapproximated solution of the system reliability, based on a discretization of the state space of the continuous variables and time space(Eymard and Mercier, 2008).Here, we employ an explicit FV scheme developed by Cocozza-Thivent et al. (CocozzaThivent, et al., 2006).The numerical scheme aims at constructing an approximate value $\rho_{t}\left(\boldsymbol{x}, \boldsymbol{y}_{i}\right) d \boldsymbol{x}$ for $p_{t}\left(d \boldsymbol{x}, \boldsymbol{y}_{i}\right)$. The estimated system reliability at time $t$, then, can be calculated as follows:

$$
\widehat{R_{F V}}(t)=\int_{\mathbf{z} \notin \mathcal{F}} \rho_{t}(\mathbf{z}) d \mathbf{z}
$$

Appendix A contains a detailed description of the FV method. Due to the complexity of the ChapmanKolmogorov equation (eq. (9)), there is no explicit expression for the variance or uncertainty associated with the estimate. However, the convergence of the method is proven in Cocozza-Thivent, et al. (2006) under the condition that $\Delta t \rightarrow 0$ and $|\mathcal{M}| / \Delta t \rightarrow 0$, where $|\mathcal{M}|$ is the space step and $\Delta t$ is the time step. The efficiency and the accuracy of the method have been shown through the numerical example in Cocozza-Thivent, et al. (2006).

\section{CASE STUDY}

We consider a subsystem of a residual heat removal system (RHRS) in a nuclear power plant, which consists of a pneumatic valve and a centrifugal pump in series shown in Fig. 4.

For the degradation model of the pump,weconsider a MSM modified from the one originally supplied by $\operatorname{EDF}($ Lin, et al., 2015a), while for the valve we take the PBM proposed inDaigle and Goebel (2011).

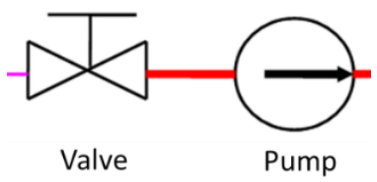

Fig. 4. Subsystem of RHRS, consisting of a centrifugal pump and a pneumatic valve.

\subsection{Centrifugal pump}

The degradation process of the pump is modeled by a four-state, continuous-time, homogeneous Markov chain as shown in Fig. 5.

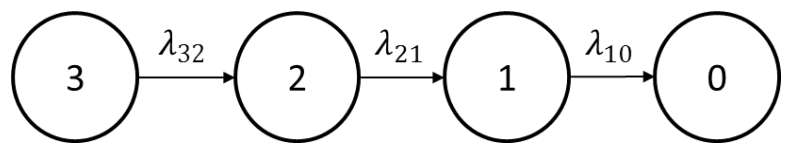


Fig. 5. Degradation process of the pump.

Among the four states of the pump, state 3 is the perfect functioning state and state 0 is the complete failure state. Let $Y_{p}(t)$ denote the degradation state of the pump at time $t$ and $\boldsymbol{S}_{p}=$ $\{3,2,1,0\}$ denote the degradation states set.The pump is functioning until $Y_{p}(t)=0$. The parameters $\lambda_{32}, \lambda_{21}$ and $\lambda_{10}$ are the transition rates between the degradation states, estimated from the available degradation and/or failure data. The pump vibrates when it reaches the degradation states 2 and 1; the intensity of the vibration of the pump on states 2 and 1 is evaluated by the experts as 'smooth' and 'rough', respectively. The set of the failure states of the pump is $\mathcal{F}_{p}=\{0\}$.

\subsection{Pneumatic valve}

The simplified scheme of the pneumatic valve is shown in Fig. 6. The degradation of the valve is the external leak at the actuator connections to the bottom pneumatic port due to corrosion, and is modeled by a PBM due to limited statistical degradation data on the valve behavior. It is much more significant than the other degradation mechanisms according to the results shown inDaigle and Goebel (2011).

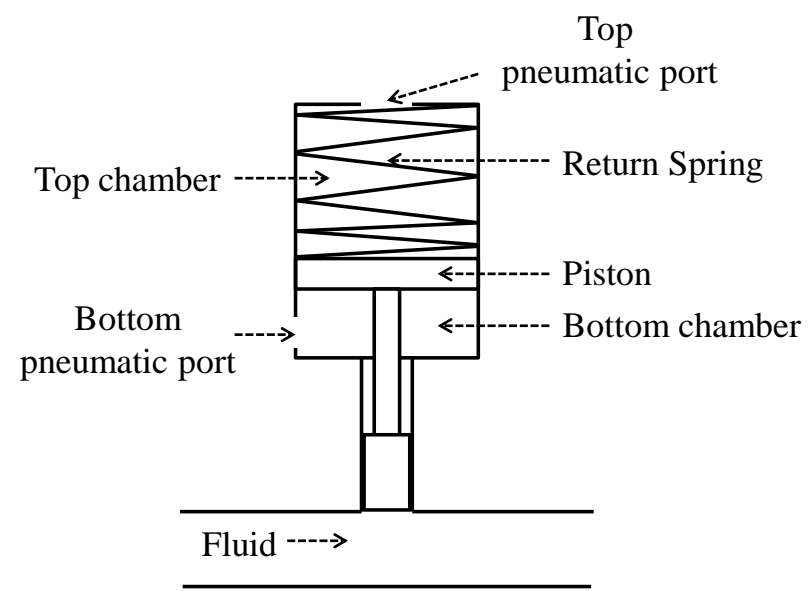

Fig.6. Simplified scheme of the pneumatic valve (Daigle and Goebel, 2011).

Let $D_{b}(t)$ denote the area of the leak hole at the bottom pneumatic port of the valve at time $t$.The development of the leak size is described by $\dot{D_{b}}(t)=\omega_{b}$, where $\omega_{b}$ is the original wear coefficient. The valve is considered failed when the size of the external leak exceeds a predefined threshold $D_{b}^{*}$. The set of the failure states of the valve is $\mathcal{F}_{v}=\left[D_{b}^{*},+\infty\right)$. 


\subsection{Dependency between degradation processes}

Dependency in the degradation processes of the two components has been indicated as a relevant problem by the experts of EDF: the pump vibrates due to degradation (Zhang, et al., 2006) which, in turn, leads the valve to vibrate, aggravating its own degradation processes (Moussou, et al., 2001).The development of the leak size of the valve is, then, reformulated as follows(Lin, et al., 2015a):

$$
\dot{D_{b}}(t)=\omega_{b}\left(1+\beta\left(Y_{p}(t)\right)\right)
$$

where $\beta\left(Y_{p}(t)\right)$ is the function indicating the relative increment of the growth rate of the external leak caused by the vibration of the pump at the degradation state $Y_{p}(t)$.

\subsection{Random shocks}

According to the experts of EDF, random shocks like water hammers and internal thermal shocks (Salonen, et al., 2007) can worsen the degradation condition of both components of the subsystem considered or even immediately lead them to failures.

Random shocks can deteriorate the pump from its currentstate $i$ to a degraded state $j$, as $p_{i j}=$ $\frac{9 \times(0.1)^{(i-j+1)}}{1-(0.1)^{(i+1)}}, i \geq j$, where $p_{i 0}$ denotes the probability of an extreme random shock leading the pump from state $i$ directly to failure state 0 .The formulation is taken fromYang, et al. (2011), which satisfies that $\sum_{j=i}^{0} p_{i j}=1$. By combining the degradation process of the pump with therandom shock process, the resultingprocesstakes the form shown in Fig. 7. The state of the process is represented by $Y(t)=$ $\left(Y_{p}(t), m\right), m \in \mathbb{N}$, where $m$ is the number of shocks experiencedby the pump.The state space of the new process is denoted by $\boldsymbol{S}=\left\{(a, b), \forall a \in \boldsymbol{S}_{p}, b \in \mathbb{N}\right\}$ andthe set of failure states of the pump is $\mathcal{F}_{\boldsymbol{p}}^{\prime}=\{(0, b), \forall b \in \mathbb{N}\}$.

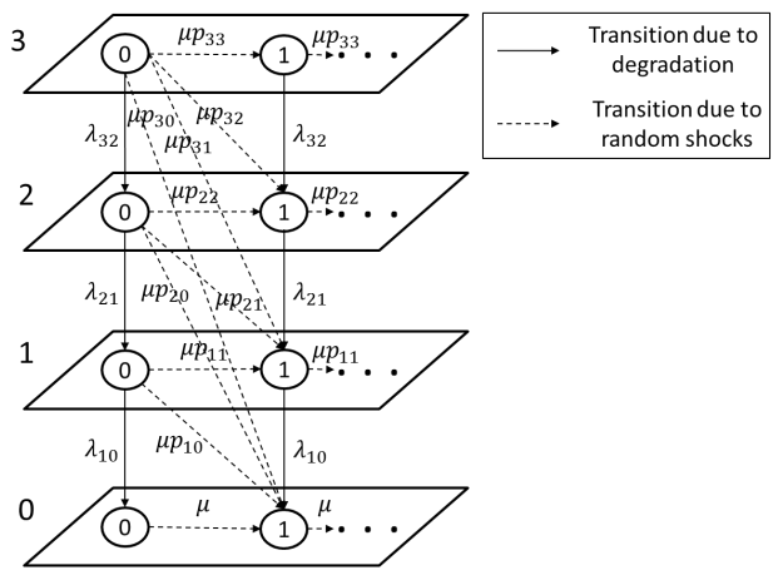

Fig. 7. Degradation and random shock processes of the pump. 
For the valve, the $i$-th shock becomes extreme if the shock load $W_{i}$ exceedsthe maximal material strength $D$,otherwise, it can bring aninstantaneous random increase $H_{i}$ to the total externalleak size(Song, et al., 2014b). Since the shock load and the damage cannot be negative, $W_{i}$ and $H_{i}$ are assumed to be i.i.d. random variables following folded normal distributions(Leone, et al., 1961), $W_{i}=$ $|a|$ and $H_{i}=|b|$, where $a \sim N\left(\mu_{h}, \sigma_{h}^{2}\right)$ and $b \sim N\left(\mu_{w}, \sigma_{w}^{2}\right)$.

\subsection{PDMP for the system considering dependency}

An illustration of the composite degradation process of the valve considering random shocks and the degradation state of the pump is shown in Fig. 8, where the system experienced a random shock at time $t_{i}$, with the shock load $W_{i}, i=1,3,4$. The first two shocks cause instantaneous random increases on $D(t)$, the last shock leads the valve to failure. The vibration of the pump accelerates the degradation process of the valve at timest $t_{2}$ and $t_{3}$, when the pump stepped to a further degraded state.The development of the leak size of the valvedoes not depend on other physical variables according to the given physics equations. Therefore, no physical variables are considered in Fig. 8 and no randomness is observed between two changing points. Nevertheless, there is actually randomness, i.e. the random changing points and the random increments. Moreover, if we consider the randomness in physical variables, then there will be randomness on the curve between two changing points.

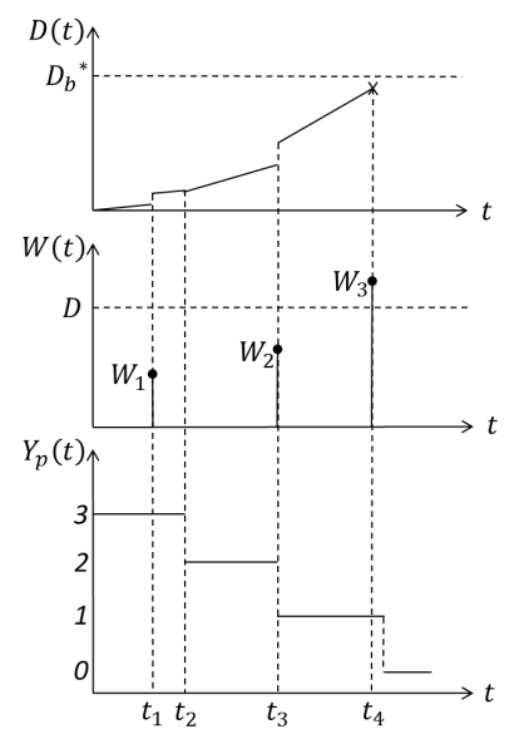

Fig. 8. An illustration of the degradation of the valveconsidering random shocks and the degradation state of the pump. Top Figure: degradation process of the valve; Center Figure: random shock processes; Bottom Figure: degradation process of the pump.

The degradation processes of the whole system can berepresented by:

$$
\boldsymbol{Z}(t)=(D(t), Y(t)) \in \mathbb{R}^{+} \times \boldsymbol{S}=\boldsymbol{E}
$$


Let $T_{k}, k \in \mathbb{N}$ denote the $k$-th jump timein $Y(t)$ and $\boldsymbol{Z}_{k}=\left(D_{k}, Y_{k}\right)=\boldsymbol{Z}\left(T_{k}\right)$. The evolution of $\boldsymbol{Z}(t)$ between two consecutive jumps of $Y(t)$, between which no shock occurs to the system and the degradation state of the pumpdoes not change, can be written as follows:

$$
\begin{aligned}
& \dot{Z}(t)=(\dot{D}(t), \dot{Y}(t)) \\
&=\left(\omega_{b}\left(1+\beta\left(Y_{p}(t)\right)\right),(0,0)\right) \\
&=(v(Y(t)),(0,0)), \text { for } t \in\left[T_{k}, T_{k+1}[(16)\right.
\end{aligned}
$$

where $v(\cdot)$ is used to denote the corresponding equation.

By integrating eq. (25), we can obtain that:

$$
\begin{aligned}
& \boldsymbol{Z}(t)=\left(D_{k}+\left(t-T_{k}\right) \omega_{b}\left(1+\beta\left(Y_{p}\left(T_{k}\right)\right)\right), Y_{k}\right) \\
&=\left(\varphi_{1}\left(\boldsymbol{Z}_{k}, t-T_{k}\right), Y_{k}\right), \text { for } t \in\left[T_{k}, T_{k+1}[\right. \\
&=\varphi\left(\boldsymbol{Z}_{k}, t-T_{k}\right), \text { for } t \in\left[T_{k}, T_{k+1}[(17)\right.
\end{aligned}
$$

where $\varphi_{1}(\cdot)$ and $\varphi(\cdot)$ are used to denote the corresponding equations.

Let $p_{t}\left(d x, \boldsymbol{y}_{i}\right)$ denote the probability distribution of $\boldsymbol{Z}(t)$. Given the series logic configuration of the system considered, the system fails when one of the two components fails; the reliability of the system at time $t$ is, then, defined as follows:

$$
R(t)=P[\boldsymbol{Z}(s) \notin \mathcal{F}, \forall s \leq t]=\int_{x \notin \mathcal{F}_{v}} \sum_{\boldsymbol{y}_{i} \notin \mathcal{F}_{\boldsymbol{p}}^{\prime}} p_{t}\left(d x, \boldsymbol{y}_{i}\right)(18)
$$

where $\mathcal{F}=\mathbb{R}^{+} \times \mathcal{F}_{\boldsymbol{p}}^{\prime} \cup \mathcal{F}_{v} \times \boldsymbol{S}$ isthe set of the failure states of the system.

The parameter values related to the system degradation processes and random shocks under accelerated aging conditions are presented in Table I. The first eight parameter values related to the degradation processes are taken fromLin, et al. (2014), the values of $\mu_{w}, \sigma_{w}$ and $D$ are taken from Peng, et al. (2010) and those of $\mu, \mu_{h}$ and $\sigma_{h}$ are assumed by expert judgment.Indeed, all parameter values are set upon the discussion with the experts from EDF.Indeed, all parameter values are set upon the discussion with the experts from EDF.

Table IParameter values

\begin{tabular}{|c|c|}
\hline Parameter & Value \\
\hline$\lambda_{32}$ & $3 \mathrm{e}-3 / \mathrm{s}$ \\
\hline$\lambda_{21}$ & $3 \mathrm{e}-3 / \mathrm{s}$ \\
\hline$\lambda_{10}$ & $3 \mathrm{e}-3 / \mathrm{s}$ \\
\hline$\omega_{b}$ & $1 \mathrm{e}-8 \mathrm{~m}^{2} / \mathrm{s}$ \\
\hline$\beta(3)$ & 0 \\
\hline$\beta(2)$ & $10 \%$ \\
\hline$\beta(1)$ & $20 \%$ \\
\hline$\beta(0)$ & 0 \\
\hline
\end{tabular}




\begin{tabular}{|c|c|}
\hline$D_{b}^{*}$ & $1.06 \mathrm{e}-5 \mathrm{~m}^{2}$ \\
\hline$\mu$ & $5 \mathrm{e}-3 / \mathrm{s}$ \\
\hline$\mu_{w}$ & $1.2 \mathrm{Gpa}$ \\
\hline$\sigma_{w}$ & $0.2 \mathrm{Gpa}$ \\
\hline$D$ & $1.5 \mathrm{Gpa}$. \\
\hline$\mu_{h}$ & $1 \mathrm{e}-7 \mathrm{~m}^{2}$ \\
\hline$\sigma_{h}$ & $2 \mathrm{e}-8 \mathrm{~m}^{2}$ \\
\hline
\end{tabular}

\section{NUMERICAL RESULTS AND ANALYSIS}

The MC simulation and the FV methods are employed to estimate the system reliability. All the experiments are carried out in MATLAB on a PC with an Intel Core 2 Duo CPU at $3.06 \mathrm{GHz}$ and a RAM of 3.07 GB. MC simulations with $10^{3}, 10^{4}$ and $10^{5}$ replications (named MC1, MC2 and MC3, respectively) are applied over a time horizon of $T_{\text {miss }}=1000 \mathrm{~s}$ for the system reliability estimation.System holding time, arrival state for stochastic process $Y(t)$ and arrival state for process $D(t)$ can be sampled by using the probability distribution eq. (28), the probability mass function eq. (30) and the probability distribution eq. (31), respectively. See Appendix B for detailed descriptions of these equations.

The results are shown in Fig. 9. It is seen that the MC simulation method requires a number of replications to achieve the desired level of accuracy. The average computation times of $\mathrm{MC1}$, MC2and MC3 are $0.21 \mathrm{~s}, 2.17 \mathrm{~s}$ and $21.77 \mathrm{~s}$, respectively.

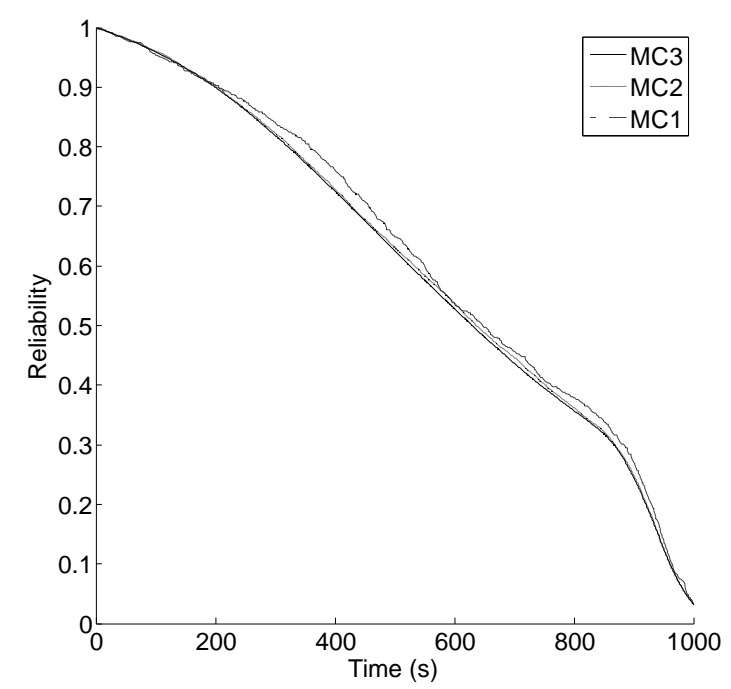

Fig. 9. System reliability estimated by MC1, MC2 and MC3.

For the FV method, the state space $\mathbb{R}^{+}$of $D(t)$ has been divided into an admissible mesh 
$\mathcal{M}=\cup_{m=0,1,2, \ldots}\left[m \Delta x,(m+1) \Delta x\left[\right.\right.$ and the time space $\mathbb{R}^{+}$has been divided into small intervals $\mathbb{R}^{+}=\bigcup_{n=0,1,2, \ldots}[n \Delta t,(n+1) \Delta t[$. See Appendix $C$ for the application of FV method.

The system reliability estimated by the FV method, is shown in Fig. 10 with the followingdifferent parameter settings: (1) FV1: $\Delta x=5 e-9, \Delta t=0.5$; (2) FV2: $\Delta x=1.5 e-$ 8, $\Delta t=1.5$ and (3) FV3: $\Delta x=4.5 e-8, \Delta t=4.5$.The accuracy of theFV scheme increases as the space step $\Delta x$ and the time step $\Delta t$ are reduced. The average computation times of FV1, FV2 and FV3 are $0.19 \mathrm{~s}, 1.93 \mathrm{~s}$ and $26.39 \mathrm{~s}$, respectively.

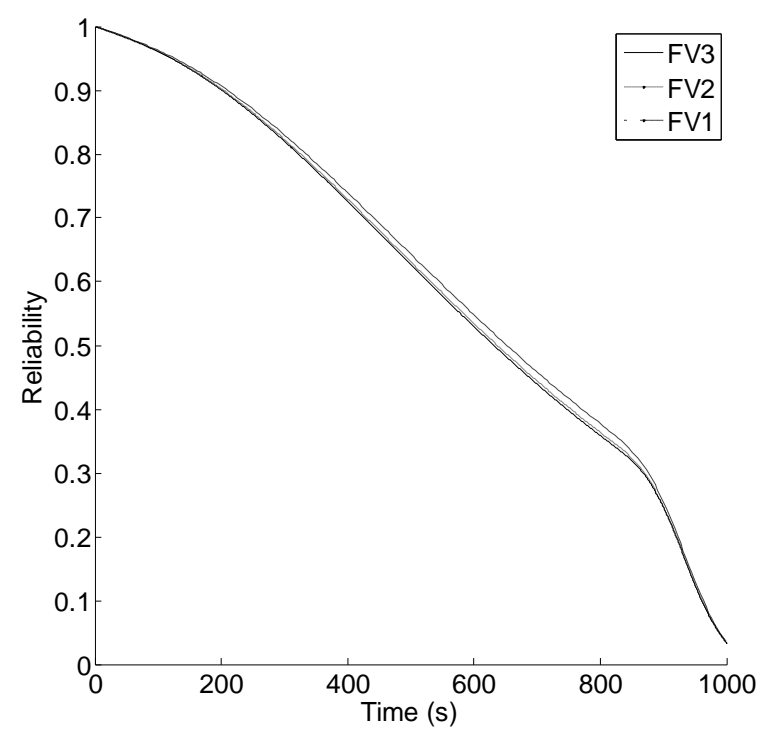

Fig. 10. System reliability estimated by FV1, FV2 and FV3.

The quantitative comparison of the most accurate results obtained by MC3 with those obtained by FV3is shown in Table II.The sample variances associated with system reliability values estimated by MC3 are less than 2.5e-6 according to eq. (12), which means the results are sufficiently consistent and accurate. The quantitative comparison of results obtained by MC3 and FV3 shown in Table II is only used to show that FV scheme can achieve comparable results to the MC simulation method (relative error less than $0.9 \%$ ) in the illustrative case. Note that FV3 gives deterministic results since the values of $\Delta x$ and $\Delta t$ do not change, which guarantees the accuracy and consistency of the quantitative comparison. To provide more information, we have added Fig. 11 to compare the results obtained by MC3 with those obtained by FV3 over the time horizon. For this case study, the computational expense of the two methods is similar.

Table IIQuantitative comparison of the results obtained by MC3 and FV3 


\begin{tabular}{|c|c|c|c|}
\hline Mime & MC3 & FV3 & $\begin{array}{c}\text { Relative } \\
\text { error }\end{array}$ \\
\hline $100 \mathrm{~s}$ & 0.9611 & 0.9607 & $0.0438 \%$ \\
\hline $200 \mathrm{~s}$ & 0.9021 & 0.9011 & $0.1162 \%$ \\
\hline $300 \mathrm{~s}$ & 0.8230 & 0.8205 & $0.3027 \%$ \\
\hline $400 \mathrm{~s}$ & 0.7285 & 0.7263 & $0.2974 \%$ \\
\hline $500 \mathrm{~s}$ & 0.6284 & 0.6271 & $0.2109 \%$ \\
\hline $600 \mathrm{~s}$ & 0.5312 & 0.5300 & $0.2394 \%$ \\
\hline $700 \mathrm{~s}$ & 0.4395 & 0.4397 & $0.0365 \%$ \\
\hline $800 \mathrm{~s}$ & 0.3576 & 0.3591 & $0.4157 \%$ \\
\hline $900 \mathrm{~s}$ & 0.2467 & 0.2459 & $0.3204 \%$ \\
\hline $1000 \mathrm{~s}$ & 0.0335 & 0.0332 & $0.8955 \%$ \\
\hline
\end{tabular}

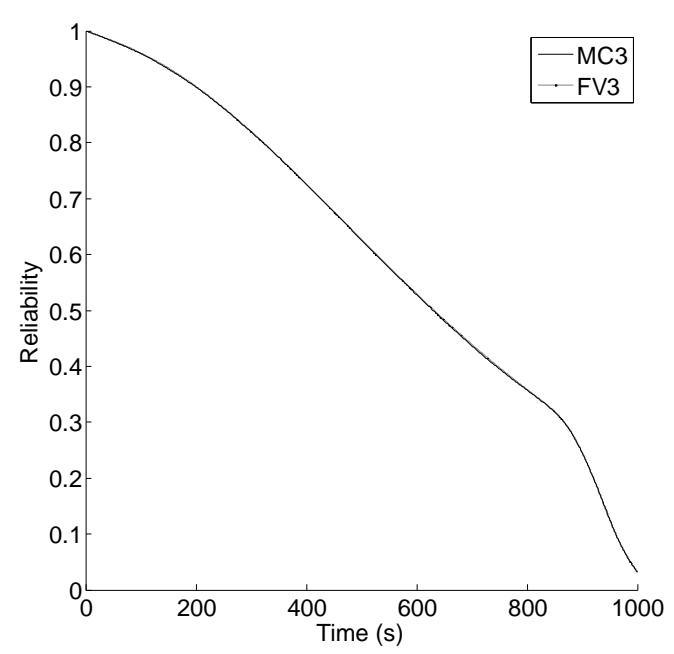

Fig. 11. Comparison of the results obtained by MC3 and FV3.

The reliability values of the valve, the pump and the system with/without random shocks, obtained by MC3, are shown in Fig. 12. The numerical comparisons on the reliability of the system, the valve and the pumpwith/without random shocks at the final time of $1000 \mathrm{~s}$ are presented in Table III. 


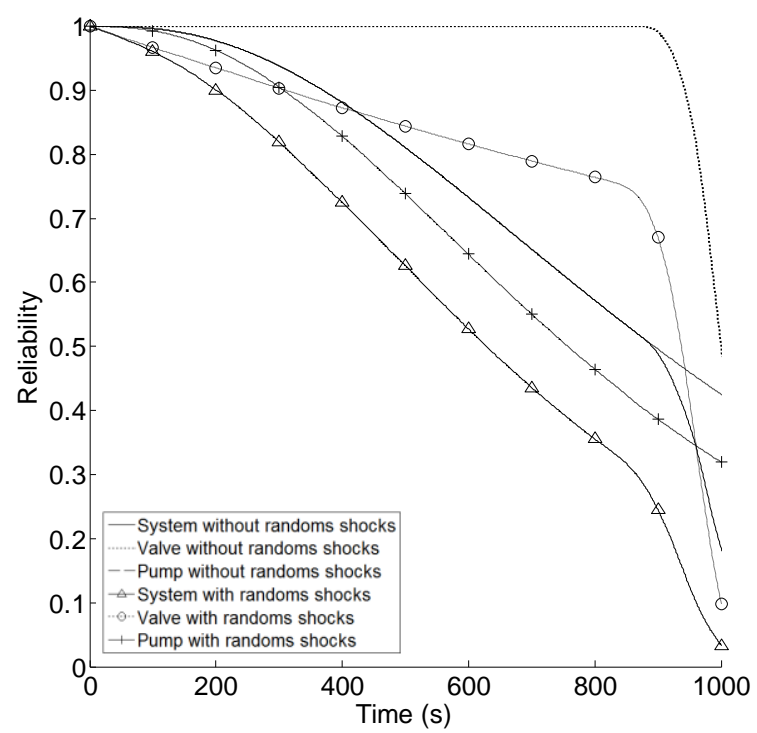

Fig. 12. The reliability of the system, the valve and the pump with/without random shocks.

When random shocks are ignored, the system reliability is basicallydetermined by the pump before around $870 \mathrm{~s}$, since the valve is highly reliable. After that, the sharp decrease of the valve reliabilitydue to degradation leads tothe same behavior in the system reliability. When random shocks are considered, the system reliability is determined by both the pump reliability and the valve reliability from the beginning until around $850 \mathrm{~s}$, since the valve is no longer as highly reliable as before. Then, the valve reliability decreases sharply due to the joint effects of random shocks and degradation, and thisdrives alsothe sharp decrease of the system reliability. We can see from the results that neglecting random shocks can result inan underestimation of the reliability of the system and of the components.

Table IIIComparison of reliabilitywith/without random shocks at $1000 \mathrm{~s}$

\begin{tabular}{|c|c|c|c|}
\hline & $\begin{array}{c}\text { Reliability without } \\
\text { random shocks }\end{array}$ & $\begin{array}{c}\text { Reliability with } \\
\text { random shocks }\end{array}$ & Relative change \\
\hline System & 0.18 & 0.033 & $81.67 \%$ \\
\hline Valve & 0.50 & 0.099 & $80.20 \%$ \\
\hline Pump & 0.43 & 0.32 & $25.58 \%$ \\
\hline
\end{tabular}

The uncertainties associated with the parameter estimates can influence the estimation of system reliability. The actual effect depends on the types and degrees of uncertainties, which are problem specific. Following one assumption of our work (i.e. limited historical data), epistemic uncertainty can arise due to the incomplete or imprecise knowledge about the degradation processes and the governing parameters of the pump and the valve, which has been considered in Lin, et al. (2015a)by 
describing the degradation model parameters as intervals (or fuzzy numbers). In the revised manuscript, we follow the settings in Lin, et al. (2015a) where a relative deviation of $\pm 10 \%$ to the original parameters values has been considered for $\lambda_{32}, \lambda_{21}, \lambda_{10}, \omega_{b}, \beta(3), \beta(2), \beta(1)$ and $\beta(0)$ upon the discussionswith the domain experts from EDF. The lower and upper bounds of system reliability under uncertainty, and the original values without uncertainty obtained by MC3 are shown in Fig. 13. The lower bound of system reliability with uncertainty decreases more sharply after around $790 \mathrm{~s}$, earlier than that without uncertainty. It is seen that the system fails after around $964 \mathrm{~s}$, because at that time the valve is completely failed. The upper bound of system reliability with uncertainty does not experience a rapid decrease because the valve is mostly functioning over the time horizon.

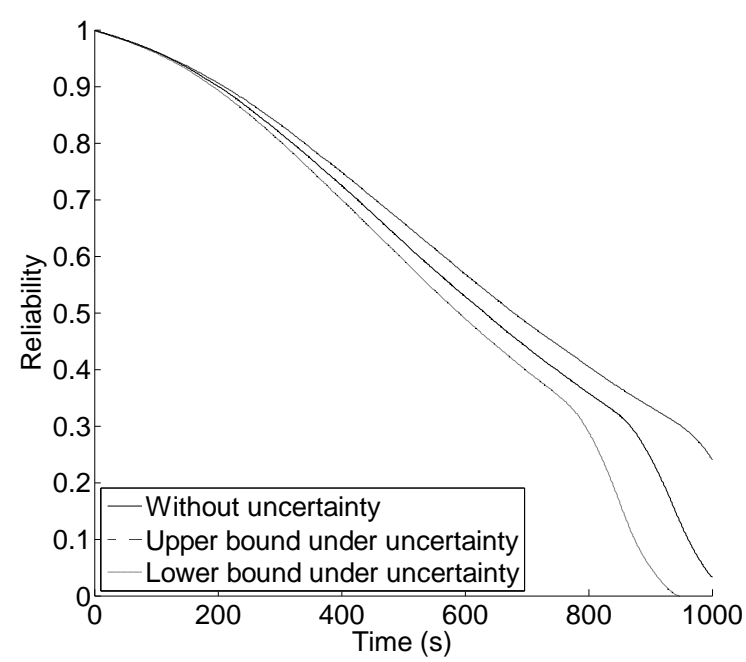

Fig. 13. The lower and upper bounds of system reliability with uncertainty, and the original values without uncertainty obtained by MC3.

\section{CONCLUSIONS}

In this paper, we presentedreliability models for systems experiencing both degradation processes and random shocks. The degradation processes involve both continuous and multi-state processes, which are modeled by MSMs and PBMs, respectively.The dependencies between degradation processes and random shocks and among degradation processes are addressed by PDMP modeling. The procedures of the MC simulation and FV methods to solve the model are developed.A subsystem of a RHRS in a nuclear power plant, which consists of a pneumatic valve and a centrifugal pump, is considered as the illustrative exampleto demonstrate the effectiveness and modeling capabilities of the proposed framework.As original contribution and differently from our previous work (Lin, et al., 2015a), this work is first in considering system reliability under both continuous and multi-state degradation processes, random shocks and their dependencies.

As future work, we will include maintenance in the model and derive optimal maintenance policies under the conditions considered. 


\section{Acknowledgement}

The authors would like to thank Mrs. Dominique VASSEUR,Mr. Antoine DESPUJOLS and Mr. Emmanuel ARDILLON from the Department Industrial Risks Management (MRI), Electricité de France R\&D for their insightful comments. The authors would like to acknowledge the financial support fromthe Electricité de France(Contract No.8610-5910112096) and the GRF project of city university of Hong Kong (GRF project No. 9042183).

\section{References}

Becker, G., Camarinopoulos, L. and Kabranis, D. (2002) Dynamic reliability under random shocks. Reliability Engineering \& System Safety, 77(3), 239-251.

Black, M., Brint, A. and Brailsford, J. (2005) A semi-Markov approach for modelling asset deterioration. Journal of the Operational Research Society,56(11), 1241-1249.

Chan, H.A. and Englert, P.J. (2001) Accelerated stress testing handbook: Guide for achieving quality products, Wiley-IEEE Press.

Chookah, M., Nuhi, M. and Modarres, M. (2011) A probabilistic physics-of-failure model for prognostic health management of structures subject to pitting and corrosion-fatigue. Reliability Engineering \& System Safety,96(12), 1601-1610.

Cocozza-Thivent, C. (2011) Processus de renouvellement markovien, Processus de Markov déterministes par morceaux. Online book available on the webpage: http://perso-math.univm/v.fr/users/cocozza.christiane/recherche-page-perso/PresentationRMetPDMP.html.

Cocozza-Thivent, C., Eymard, R. and Mercier, S. (2006) A finite-volume scheme for dynamic reliability models. IMA journal of numerical analysis,26(3), 446-471.

Daigle, M.J. and Goebel, K. (2011) A model-based prognostics approach applied to pneumatic valves. International Journal of Prognostics and Health Management, 2(2), 84-99.

Daigle, M.J. and Goebel, K. (2013) Model-based prognostics with concurrent damage progression processes. Systems, Man, and Cybernetics: Systems, IEEE Transactions on 43(3), 535-546.

Davis, M.H. (1993) Markov Models \& Optimization, CRC Press.

Eymard, R. and Mercier, S. (2008) Comparison of numerical methods for the assessment of production availability of a hybrid system. Reliability Engineering \& System Safety,93(1), 168-177.

Giorgio, M., Guida, M. and Pulcini, G. (2011) An age-and state-dependent Markov model for degradation processes. IIE Transactions,43(9), 621-632.

Hu, X., Xiong, R. and Egardt, B. (2014) Model-based dynamic power assessment of lithium-ion batteries considering different operating conditions. Industrial Informatics, IEEE Transactions on,10(3), 1948-1959.

Jiang, L., Feng, Q. and Coit, D.W. (2012) Reliability and maintenance modeling for dependent competing failure processes with shifting failure thresholds. Reliability, IEEE Transactions on,61(4), 932-948. 
Jiang, L., Feng, Q. and Coit, D.W. (2015) Modeling zoned shock effects on stochastic degradation in dependent failure processes. IIE Transactions,47(5), 460-470.

Keedy, E. and Feng, Q. (2013) Reliability analysis and customized preventive maintenance policies for stents with stochastic dependent competing risk processes. Reliability, IEEE Transactions on,62(4), 887-897.

Labeau, P.-E. (1996) A Monte Carlo estimation of the marginal distributions in a problem of probabilistic dynamics. Reliability Engineering \& System Safety,52(1), 65-75.

Leone, F., Nelson, L. and Nottingham, R. (1961) The folded normal distribution. Technometrics,3(4), 543-550.

Lewis, E. and Böhm, F. (1984) Monte Carlo simulation of Markov unreliability models. Nuclear engineering and design,77(1), 49-62.

Lin, Y.-H., Li, Y.-F. and Zio, E. (2015a) Fuzzy Reliability Assessment of Systems with Multiple Dependent Competing Degradation Processes. Fuzzy systems, IEEE Transactions on,23(5), 1428-1438.

Lin, Y.-H., Li, Y.-F. and Zio, E. (2015b) Integrating Random Shocks Into Multi-State Physics Models of Degradation Processes for Component Reliability Assessment. Reliability, IEEE Transactions on,64(1), 154-166.

Lin, Y.-H., Li, Y. and Zio, E. (2014) Dynamic Reliability Models for Multiple Dependent Competing Degradation Processes. Proceedings of ESREL 2014, 775-782.

Lisnianski, A., Frenkel, I. and Ding, Y. (2010) Statistical Analysis of Reliability Data for Multi-state Systems. Multistate System Reliability Analysis and Optimization for Engineers and Industrial Managers, 117-142.

Lorton, A., Fouladirad, M. and Grall, A. (2013) A methodology for probabilistic model-based prognosis. European Journal of Operational Research,225(3), 443-454.

Marseguerra, M. and Zio, E. (1996) Monte Carlo approach to PSA for dynamic process systems. Reliability Engineering \& System Safety,52(3), 227-241.

Marseguerra, M., Zio, E. and Podofillini, L. (2004) A multiobjective genetic algorithm approach to the optimization of the technical specifications of a nuclear safety system. Reliability Engineering \& System Safety,84(1), 87-99.

Moghaddass, R. and Zuo, M.J. (2014) Multistate degradation and supervised estimation methods for a condition-monitored device. IIE Transactions,46(2), 131-148.

Moussou, P., Cambier, S., Lachene, D., Longarini, S., Paulhiac, L. and Villouvier, V. (2001) Vibration investigation of a French PWR power plant piping system caused by cavitating butterfly valves. ASME-PUBLICATIONSPVP,420(99-106.

Newby, M. (1991) Estimation of Paris-Erdogan law parameters and the influence of environmental factors on crack growth. International journal of fatigue,13(4), 291-301.

Ogurtsova, E. (2014) Estimating transition rates for multistate models from panel data and repeated crosssections. University of Groningen.

Peng, H., Feng, Q. and Coit, D.W. (2010) Reliability and maintenance modeling for systems subject to multiple dependent competing failure processes. IIE Transactions,43(1), 12-22.

Rafiee, K., Feng, Q. and Coit, D.W. (2014) Reliability modeling for dependent competing failure processes with changing degradation rate. IIE Transactions,46(5), 483-496.

Rasmekomen, N. and Parlikad, A.K. (2013) Maintenance optimization for asset systems with dependent 
performance degradation. Reliability, IEEE Transactions on,62(2), 362-367.

Ruiz-Castro, J.E. (2016) Markov counting and reward processes for analysing the performance of a complex system subject to random inspections. Reliability Engineering \& System Safety,145(155-168.

Salonen, J., Auerkari, P., Lehtinen, O. and Pihkakoski, M. (2007) Experience on in-service damage in power plant components. Engineering Failure Analysis,14(6), 970-977.

Schöttl, A. (1996) A reliability model of a system with dependent components. IEEE transactions on reliability,45(2), 267-271.

Song, S., Coit, D.W. and Feng, Q. (2014a) Reliability for systems of degrading components with distinct component shock sets. Reliability Engineering \& System Safety,132(115-124.

Song, S., Coit, D.W., Feng, Q. and Peng, H. (2014b) Reliability analysis for multi-component systems subject to multiple dependent competing failure processes. Reliability, IEEE Transactions on,63(1), 331-345.

Wang, W., Shen, Z. and Dinavahi, V. (2014) Physics-Based Device-Level Power Electronic Circuit Hardware Emulation on FPGA. Industrial Informatics, IEEE Transactions on,10(4), 2166-2179.

Wang, Y. and Pham, H. (2012) Modeling the dependent competing risks with multiple degradation processes and random shock using time-varying copulas. Reliability, IEEE Transactions on,61(1), 13-22.

Wang, Z., Huang, H.-Z., Li, Y. and Xiao, N.-C. (2011) An approach to reliability assessment under degradation and shock process. Reliability, IEEE Transactions on,60(4), 852-863.

Yang, W.S., Lim, D.E. and Chae, K.C. (2011) Maintenance of multi-state production systems deteriorated by random shocks and production. Journal of Systems Science and Systems Engineering,20(1), 110-118.

Ye, Z.S., Tang, L.C. and Xu, H.Y. (2011) A distribution-based systems reliability model under extreme shocks and natural degradation. Reliability, IEEE Transactions on,60(1), 246-256.

Yeh, R.H. (1997) Optimal inspection and replacement policies for multi-state deteriorating systems. European Journal of Operational Research,96(2), 248-259.

Zhang, S., Hodkiewicz, M., Ma, L. and Mathew, J. (2006) Machinery condition prognosis using multivariate analysis, in Engineering asset management, Springer, pp. 847-854.

Zio, E. (2013) The Monte Carlo simulation method for system reliability and risk analysis, Springer.

Zuo, M.J., Liu, B. and Murthy, D. (2000) Replacement-repair policy for multi-state deteriorating products under warranty. European Journal of Operational Research,123(3), 519-530.

Zwillinger, D. (1998) Handbook of differential equations, Gulf Professional Publishing.

\section{Appendix A: FV method}

\section{Assumptions}

The FV method for determining the approximated solution of the system reliability can be developed under the following assumptions (Cocozza-Thivent, et al., 2006): 
- The transition rates $\lambda_{\boldsymbol{y}_{i}, \boldsymbol{y}_{j}}\left(\cdot \mid \boldsymbol{\theta}_{\boldsymbol{K}}\right), \forall \boldsymbol{y}_{i}, \boldsymbol{y}_{j} \in \boldsymbol{S}^{\prime}$ are continuous and bounded functions from $\mathbb{R}^{d_{L}}$ to $\mathbb{R}^{+}$.

- The physics equations $\boldsymbol{f}_{\boldsymbol{L}} \boldsymbol{y}_{i}\left(\cdot \mid \boldsymbol{\theta}_{\boldsymbol{L}}\right), \forall \boldsymbol{y}_{j} \in \boldsymbol{S}^{\prime}$ are continuous functions from $\mathbb{R}^{d_{L}}$ to $\mathbb{R}^{d_{L}}$ and locally Lipschitz continuous.

- The physics equations $\boldsymbol{f}_{\boldsymbol{L}}^{\boldsymbol{y}_{i}}\left(\cdot \mid \boldsymbol{\theta}_{\boldsymbol{L}}\right), \forall \boldsymbol{y}_{i} \in \boldsymbol{S}^{\prime}$ are sub-linear, i.e. there are some $V_{1}>0$ and $V_{2}>0$ such that

$$
\forall \boldsymbol{x} \in \mathbb{R}^{d_{L}}, t \in \mathbb{R}^{+}\left|\boldsymbol{f}_{\boldsymbol{L}}^{\boldsymbol{y}_{i}}\left(\boldsymbol{x} \mid \boldsymbol{\theta}_{\boldsymbol{L}}\right)\right| \leq V_{1}\|\boldsymbol{x}\|+V_{2}
$$

- The functions $\operatorname{div}\left(\boldsymbol{f}_{\boldsymbol{L}}^{\boldsymbol{y}_{i}}\left(\cdot \mid \boldsymbol{\theta}_{\boldsymbol{L}}\right)\right), \forall \boldsymbol{y}_{i} \in \boldsymbol{S}^{\prime}$ are almost everywhere bounded in absolute value by some real value $D>0$ (independent of $\boldsymbol{y}_{i}$ ).

- If $\phi(\cdot)$ is a continuous and bounded function from $\mathbb{R}^{d_{L}}$ to $\mathbb{R}$, then, $\boldsymbol{x} \rightarrow \int \phi(\vec{y}) \mu\left(\boldsymbol{y}_{i}, \boldsymbol{y}_{j}, \boldsymbol{x}\right)(d \boldsymbol{y})$ is continuous from $\mathbb{R}^{d_{L}}$ to $\mathbb{R}$.

\section{Solution approach}

For ease of notation, we let $\boldsymbol{g}^{\boldsymbol{y}_{i}}\left(\cdot, \cdot \mid \boldsymbol{\theta}_{\boldsymbol{L}}\right): \mathbb{R}^{d_{L}} \times \mathbb{R} \rightarrow \mathbb{R}^{d_{L}}$ denote the solution of

$$
\frac{\partial}{\partial t} \boldsymbol{g}^{\boldsymbol{y}_{i}}\left(\boldsymbol{x}, t \mid \boldsymbol{\theta}_{\boldsymbol{L}}\right)=\boldsymbol{f}_{\boldsymbol{L}}^{\boldsymbol{y}_{i}}\left(\boldsymbol{g}^{\boldsymbol{y}_{i}}\left(\boldsymbol{x}, t \mid \boldsymbol{\theta}_{\boldsymbol{L}}\right) \mid \boldsymbol{\theta}_{\boldsymbol{L}}\right), \forall \boldsymbol{y}_{i} \in \boldsymbol{S}^{\prime}, \boldsymbol{x} \in \mathbb{R}^{d_{L}}, t \in \mathbb{R}
$$

with

$$
\boldsymbol{g}^{\boldsymbol{y}_{i}}\left(\boldsymbol{x}, 0 \mid \boldsymbol{\theta}_{\boldsymbol{L}}\right)=\boldsymbol{x}, \forall \boldsymbol{y}_{i} \in \boldsymbol{S}^{\prime}, x \in \mathbb{R}^{\mathrm{d}_{\mathrm{L}}}
$$

and $\boldsymbol{g}^{\boldsymbol{y}_{i}}\left(\boldsymbol{x}, t \mid \boldsymbol{\theta}_{\boldsymbol{L}}\right)$ being the result of the deterministic behavior of $\boldsymbol{X}(t)$ after time $t$, starting from the point $\boldsymbol{x}$ while the processes $\boldsymbol{Y}^{\prime}(t)$ hold on state $\boldsymbol{y}_{i}$.

The state space $\mathbb{R}^{d_{L}}$ of continuous variables $\boldsymbol{X}^{\prime}(t)$ is divided into an admissible mesh $\mathcal{M}$, which is a family of measurable subsets of $\mathbb{R}^{d_{L}}$, i.e., $\mathcal{M}$ is a partition of $\mathbb{R}^{d_{L}}$ such that:

(1) $\cup_{A \in \mathcal{M}} A=\mathbb{R}^{d_{L}}$.

(2) $\forall A, B \in \mathcal{M}, A \neq B \Rightarrow A \cap B=\emptyset$.

(3) $m_{A}=\int_{A} d \boldsymbol{x}>0, \forall A \in \mathcal{M}$, where $m_{A}$ is the volume of grid $A$.

(4) $\sup _{A \in \mathcal{M}} \operatorname{diam}(A)<+\infty$ where $\operatorname{diam}(A)=\sup _{\forall \boldsymbol{x}, \boldsymbol{y} \in A}|\boldsymbol{x}-\boldsymbol{y}|$.

Additionally, the time space $\mathbb{R}^{+}$is divided into small intervals $\mathbb{R}^{+}=\bigcup_{n=0,1,2, \ldots}[n \Delta t,(n+1) \Delta t$ [ by setting the time step $\Delta t>0$ (the length of each interval).

The numerical scheme aims at constructing an approximate value $\rho_{t}\left(\boldsymbol{x},{ }^{\cdot}\right) d \boldsymbol{x}$ for $p_{t}\left(d \boldsymbol{x},{ }^{\cdot}\right)$, such that $\rho_{t}(\boldsymbol{x}, \cdot)$ is constant on each $\left[n \Delta t,(n+1) \Delta t\left[\times A \times\left\{\boldsymbol{y}_{i}\right\}, \forall A \in \mathcal{M}, \boldsymbol{y}_{i} \in \boldsymbol{S}^{\prime}\right.\right.$ :

$$
\rho_{t}\left(\boldsymbol{x}, \boldsymbol{y}_{i}\right)=P_{n}\left(A, \boldsymbol{y}_{i}\right), \forall \boldsymbol{y}_{i} \in \boldsymbol{S}^{\prime}, \boldsymbol{x} \in A, t \in[n \Delta t,(n+1) \Delta t[
$$

$P_{0}\left(A, \boldsymbol{y}_{i}\right), \forall \boldsymbol{y}_{i} \in \boldsymbol{S}^{\prime}, A \in \mathcal{M}$ is defined as follows:

$$
P_{0}\left(A, \boldsymbol{y}_{i}\right)=\int_{A} p_{0}\left(d \boldsymbol{x}, \boldsymbol{y}_{i}\right) / m_{A}
$$

Then, $P_{n+1}\left(A, \boldsymbol{y}_{i}\right)$ can be calculated considering the deterministic evaluation of $\boldsymbol{X}(t)$ and the stochastic evolution of $\boldsymbol{Y}^{\prime}(t)$ based on $P_{n}\left(\mathcal{M}, \boldsymbol{y}_{i}\right)$ by the Chapman-Kolmogorov forward equation, as 
follows:

$$
\begin{gathered}
P_{n+1}\left(A, \boldsymbol{y}_{i}\right) \\
=\frac{1}{1+\Delta t b_{A}^{\boldsymbol{y}_{i}}} \widehat{P_{n+1}}\left(A, \boldsymbol{y}_{i}\right)+\Delta t \sum_{B \in \mathcal{M}} \sum_{\boldsymbol{y}_{j} \in \boldsymbol{s}^{\prime}} \frac{a_{B, A}^{\boldsymbol{y}_{j}, \boldsymbol{y}_{i}}}{1+\Delta t b_{A}^{\boldsymbol{y}_{j}}} \widehat{P_{n+1}}\left(B, \boldsymbol{y}_{j}\right)
\end{gathered}
$$

where

$$
a_{B, A}^{\boldsymbol{y}_{j}, \boldsymbol{y}_{i}}=\int_{A} \lambda_{\boldsymbol{y}_{j}, \boldsymbol{y}_{i}}\left(\boldsymbol{x} \mid \boldsymbol{\theta}_{\boldsymbol{K}}\right) \int_{B} \mu\left(\boldsymbol{y}_{j}, \boldsymbol{y}_{i}, \boldsymbol{x}\right)(d \boldsymbol{y}) \boldsymbol{x} / m_{A}
$$

is the average transition rate from state $\boldsymbol{y}_{j}$ and grid $B$ to state $\boldsymbol{y}_{i}$ and grid $A$,

$$
b_{A}^{\boldsymbol{y}_{i}}=\int_{A} \sum_{\boldsymbol{y}_{j} \in \boldsymbol{s}^{\prime}} \lambda_{\boldsymbol{y}_{i}, \boldsymbol{y}_{j}}\left(\boldsymbol{x} \mid \boldsymbol{\theta}_{\boldsymbol{K}}\right) d \boldsymbol{x} / m_{A}
$$

is the average transition rate out of state $\boldsymbol{y}_{i}$ for grid $A$,

$$
\widehat{P_{n+1}}\left(A, \boldsymbol{y}_{i}\right)=\sum_{B \in \mathcal{M}} m_{B A}^{\boldsymbol{y}_{i}} P_{n}\left(B, \boldsymbol{y}_{i}\right) / m_{A}
$$

is the approximate value of probability density function on $\left[(n+1) \Delta t,(n+2) \Delta t\left[\times A \times\left\{\boldsymbol{y}_{i}\right\}\right.\right.$ according to the deterministic evolution of $\boldsymbol{X}(t)$,

$$
m_{B A}^{\boldsymbol{y}_{i}}=\int_{\left\{\boldsymbol{y} \in B \mid \boldsymbol{g}^{\boldsymbol{y}_{i}}\left(\boldsymbol{y}, \Delta t \mid \boldsymbol{\theta}_{\mathbf{L}}\right) \in A\right\}} d \boldsymbol{y}
$$

is the volume of the part of grid $B$ which will enter grid $A$ after time $\Delta t$, according to the deterministic evolution of $\boldsymbol{X}(t)$.

The approximated solution $\rho_{t}(\boldsymbol{x}, \cdot) d \boldsymbol{x}$ weakly converges towards $p_{t}(d \boldsymbol{x}, \cdot)$ when $\Delta t \rightarrow 0$ and $|\mathcal{M}| /$ $\Delta t \rightarrow 0$ where $|\mathcal{M}|=\sup _{A \in \mathcal{M}} \operatorname{diam}(A)$.

\section{Appendix B: Equations for MC simulation method in case study}

The semi-Markov kernel of $\left\{\boldsymbol{Z}_{n}, T_{n}\right\}_{n \geq 0}$ is $\left(\boldsymbol{i}=\left(x, \boldsymbol{y}_{i}\right),\left(d x, \boldsymbol{y}_{j}\right), d t\right)=Q\left(\varphi(\boldsymbol{i}, t),\left(d x, \boldsymbol{y}_{j}\right)\right) d F_{\boldsymbol{i}}(t), \forall k \in \mathbb{N}, \boldsymbol{y}_{i}, \boldsymbol{y}_{j} \in \boldsymbol{S}, x \in \mathbb{R}^{+}, d x \rightarrow 0, d t \rightarrow 0$. According to the degradation models of the system, we can obtain that:

$$
d F_{\boldsymbol{i}=\left(x, \boldsymbol{y}_{i}\right)}(t)=\lambda_{\boldsymbol{y}_{i}} e^{-\lambda_{\boldsymbol{y}_{i}} t} d t(28)
$$

where $\lambda_{\boldsymbol{y}_{i}}$ is the sum of the outgoing transition rates of $Y(t)$ from state $\boldsymbol{y}_{i}$, and

$$
\begin{gathered}
Q\left(\varphi(\boldsymbol{i}, t),\left(d x, \boldsymbol{y}_{i}\right)\right) \\
=P\left[D_{k+1} \in[x, x+d x] \mid Y_{k+1}=\boldsymbol{y}_{j}, T_{k+1}-T_{k} \in[t, t+d t], \boldsymbol{Z}_{k}=\boldsymbol{i}\right] \\
\cdot P\left[Y_{k+1}=\boldsymbol{y}_{j} \mid T_{k+1}-T_{k} \in[t, t+d t], \boldsymbol{Z}_{k}=\boldsymbol{i}\right]
\end{gathered}
$$

where

$$
\begin{aligned}
P\left[Y_{k+1}=\right. & \left.\boldsymbol{y}_{j} \mid T_{k+1}-T_{k} \in[t, t+d t], \boldsymbol{Z}_{k}=\boldsymbol{i}\right] \\
& =P\left[Y_{k+1}=\boldsymbol{y}_{j} \mid Y_{k}=\boldsymbol{y}_{i}\right]
\end{aligned}
$$$$
=\frac{\lambda_{y_{i}, y_{j}}}{\lambda_{y_{i}}}(30)
$$

where $\lambda_{\boldsymbol{y}_{i}, \boldsymbol{y}_{j}}$ is the transition rate of $Y(t)$ from state $\boldsymbol{y}_{i}$ to state $\boldsymbol{y}_{j}$, and 


$$
\begin{array}{r}
P\left[D_{k+1} \in[x, x+d x] \mid Y_{k+1}=\boldsymbol{y}_{j}, T_{k+1}-T_{k} \in[t, t+d t], \boldsymbol{Z}_{k}=\boldsymbol{i}\right] \\
P\left[\varphi_{1}(\boldsymbol{i}, t)+H\left(T_{k}+t\right) \in[x, x+d x]\right], \\
=\left\{\begin{array}{r}
\text { if transition from } \boldsymbol{y}_{i} \text { to } \boldsymbol{y}_{j} \text { is due to random shock } \\
\delta_{\varphi_{1}(i, t)}(d x), \\
\text { if transition from } \boldsymbol{y}_{i} \text { to } \boldsymbol{y}_{j} \text { is due to degradation }
\end{array}\right.
\end{array}
$$

where $H\left(T_{k}+t\right)$ is the instantaneous random increase caused by shock at time $T_{k}+t, \delta$ is the Dirac delta function and

$$
\begin{gathered}
P\left[\varphi_{1}(\boldsymbol{i}, t)+H\left(T_{k}+t\right) \in[x, x+d x]\right] \\
\left.\Phi\left(\frac{D-\mu_{w}}{\sigma_{w}}\right) \cdot \frac{1}{\sigma_{h}} \phi\left(\frac{x-\varphi_{1}(i, t)-\mu_{h}}{\sigma_{h}}\right)\right) d x \\
\text { if } x<D_{b}^{*} \\
\left(1-\Phi\left(\frac{D-\mu_{w}}{\sigma_{w}}\right)\right) \cdot \delta_{\varphi_{1}(i, t)+D_{b}^{*}}\left(\text { dx } x+\Phi\left(\frac{D-\mu_{w}}{\sigma_{w}}\right) \cdot \frac{1}{\sigma_{h}} \phi\left(\frac{x-\varphi_{1}(i, t)-\mu_{h}}{\sigma_{h}}\right)\right) d x \\
\text { if } x \geq D_{b}^{*}
\end{gathered}
$$

where $\Phi(\cdot)$ and $\phi(\cdot)$ are the cumulative distribution function and the probability density function of a folded normal distribution related to the standard normal distribution, respectively. Here, since an extreme shock can directly lead the valve to failure, we assume each extreme shock increase the total external leak size by $D_{b}^{*}$ to formulate the problem within the settings of PDMP. Note that this assumption will not change the reliability of the valve.

\section{Appendix C: Application of FV method in case study}

The probability distribution of $\boldsymbol{Z}(t), p_{t}\left(d x, \boldsymbol{y}_{i}\right)$, obeys the Chapman-Kolmogorov equation (Davis, 1993) as follows:

$$
\begin{gathered}
\int_{0}^{t} \sum_{\boldsymbol{y}_{i} \in \boldsymbol{S}} \int_{\mathbb{R}^{+}} \sum_{\boldsymbol{y}_{j} \in \boldsymbol{S}} \lambda_{\boldsymbol{y}_{i}, \boldsymbol{y}_{j}}\left(\int_{\mathbb{R}^{+}} \psi\left(\boldsymbol{y}_{j}, y\right) \mu\left(\boldsymbol{y}_{i}, \boldsymbol{y}_{j}, x\right)(d y)-\psi\left(\boldsymbol{y}_{i}, x\right)\right) p_{s}\left(d x, \boldsymbol{y}_{i}\right) d s+ \\
\int_{0}^{t} \sum_{\boldsymbol{y}_{i} \in \boldsymbol{S}} \int_{\mathbb{R}^{+}} v\left(\boldsymbol{y}_{i}\right) \operatorname{div}\left(\psi\left(\boldsymbol{y}_{i}, x\right)\right) p_{s}\left(d x, \boldsymbol{y}_{i}\right) d s-\sum_{\boldsymbol{y}_{i} \in S} \int_{\mathbb{R}^{+}} \psi\left(\boldsymbol{y}_{i}, x\right) p_{t}\left(d x, \boldsymbol{y}_{i}\right)+ \\
\sum_{\boldsymbol{y}_{i} \in \boldsymbol{S}} \int_{\mathbb{R}^{+}} \psi\left(\boldsymbol{y}_{i}, x\right) p_{0}\left(d x, \boldsymbol{y}_{i}\right)=0
\end{gathered}
$$

where $\psi(\because$,$) is any continuously differentiable function from \boldsymbol{S} \times \mathbb{R}^{+}$to $\mathbb{R}$ with a compact support and $\mu\left(\boldsymbol{y}_{i}, \boldsymbol{y}_{j}, x\right)(d y)$ is the probability of $D(t) \in[y, y+d y]$ after jumping from $x$ when $Y(t)$ steps to state $\boldsymbol{y}_{j}$ from state $\boldsymbol{y}_{i}$ as follows: 


$$
\mu\left(\boldsymbol{y}_{i}, \boldsymbol{y}_{j}, x\right)(d y)=\left\{\begin{array}{c}
\left.\Phi\left(\frac{D-\mu_{w}}{\sigma_{w}}\right) \cdot \frac{1}{\sigma_{h}} \phi\left(\frac{y-x-\mu_{h}}{\sigma_{h}}\right)\right) d y, \\
\text { if transition from } \boldsymbol{y}_{i} \text { to } \boldsymbol{y}_{j} \text { is due to random shock and } y<D_{b}^{*} \\
\left.\left(1-\Phi\left(\frac{D-\mu_{w}}{\sigma_{w}}\right)\right) \cdot \delta_{x+D_{b}^{*}}(d y)+\Phi\left(\frac{D-\mu_{w}}{\sigma_{w}}\right) \cdot \frac{1}{\sigma_{h}} \phi\left(\frac{y-x-\mu_{h}}{\sigma_{h}}\right)\right) d y \\
\text { if transition from } \boldsymbol{y}_{i} \text { to } \boldsymbol{y}_{j} \text { is due to random shock and } y \geq D_{b}^{*} \\
\quad \delta_{x}(d y), \\
\text { if transition from } \boldsymbol{y}_{i} \text { to } \boldsymbol{y}_{j} \text { is due to degradation }
\end{array}\right.
$$

$P_{0}\left(m, \boldsymbol{y}_{i}\right)$ is defined as follows:

$$
P_{0}\left(m, \boldsymbol{y}_{i}\right)=\int_{m \Delta x}^{(m+1) \Delta x} p_{0}\left(d x, \boldsymbol{y}_{i}\right) / \Delta x
$$

where $p_{0}\left(d x, \boldsymbol{y}_{i}\right)=\delta_{0}(d x) \cdot \mathbf{1}_{\left\{\boldsymbol{y}_{i}=(3,0)\right\}}$. Then, $P_{n+1}\left(m, \boldsymbol{y}_{i}\right), n \in \mathbb{N}$ can be calculated considering the deterministic evolution of $D(t)$ and the stochastic evolution of $Y(t)$ based on $P_{n}(\cdot, \cdot)$ by the Chapman-Kolmogorov forward equation, as follows:

$$
\begin{gathered}
P_{n+1}\left(m, \boldsymbol{y}_{i}\right) \\
=\frac{1}{1+\Delta t \lambda_{\boldsymbol{y}_{i}}} \widehat{P_{n+1}}\left(m, \boldsymbol{y}_{i}\right)+\Delta t \sum_{m^{\prime} \in \mathbb{N}} \sum_{\boldsymbol{y}_{j} \in \boldsymbol{S}} \frac{a^{\boldsymbol{y}_{j},{ }^{\prime}, m}}{1+\Delta t \lambda_{\boldsymbol{y}_{j}}} \widehat{P_{n+1}}\left(m^{\prime}, \boldsymbol{y}_{j}\right)
\end{gathered}
$$

where

$$
a_{m^{\prime}, m}^{\boldsymbol{y}_{j}, \boldsymbol{y}_{i}}=\lambda_{\boldsymbol{y}_{j}, \boldsymbol{y}_{i}} \int_{m^{\prime} \Delta x}^{\left(m^{\prime}+1\right) \Delta x} \int_{m \Delta x}^{(m+1) \Delta x} \mu\left(\boldsymbol{y}_{j}, \boldsymbol{y}_{i}, x\right)(d y) d x / \Delta x
$$

is the average transition rate from state $\boldsymbol{y}_{j}$ and grid $\left[m^{\prime} \Delta x,\left(m^{\prime}+1\right) \Delta x\right.$ [ to state $\boldsymbol{y}_{i}$ and grid $[m \Delta x,(m+1) \Delta x[$

$$
\widehat{P_{n+1}}\left(m, \boldsymbol{y}_{i}\right)=\sum_{m^{\prime} \in \mathbb{N}} v_{m, m}^{\boldsymbol{y}_{i}{ }^{\prime}, m} P_{n}\left(m^{\prime}, \boldsymbol{y}_{i}\right) / \Delta x
$$

is the approximate value of probability density function on $\left[m \Delta x,(m+1) \Delta x\left[\times\left\{\boldsymbol{y}_{i}\right\}\right.\right.$ according to the deterministic evolution of $D(t)$ between jumps of $Y(t)$ and

$$
v_{m^{\prime}, m}^{\boldsymbol{y}_{i^{\prime}}}=\int_{\left\{x \in \left[m^{\prime} \Delta x,\left(m^{\prime}+1\right) \Delta x\left[I \left(\varphi_{1}\left(\left(x, y_{i}\right), \Delta t\right) \in[m \Delta x,(m+1) \Delta x[\}\right.\right.\right.\right.} d x
$$

is the volume of the part of grid $\left[m^{\prime} \Delta x,\left(m^{\prime}+1\right) \Delta x\right.$ [ which will enter grid $[m \Delta x,(m+1) \Delta x[$ after time $\Delta t$ according to the deterministic evaluation of $D(t)$. 